\title{
Selective Synthesis of Functionalized Thia- and Oxacalix[2]arene[2]pyrimidines
}

Wouter Maes, ${ }^{\dagger}$ Wim Van Rossom, ${ }^{\dagger}$ Kristof Van Hecke,${ }^{\ddagger}$ Luc Van Meervelt, ${ }^{\ddagger}$ and Wim Dehaen ${ }^{*}{ }^{\dagger}$

${ }^{\dagger}$ Molecular Design and Synthesis, Department of Chemistry, Katholieke Universiteit Leuven, Celestijnenlaan 200F, B3001 Leuven, Belgium.

‡ Biomolecular Architecture, Department of Chemistry, Katholieke Universiteit Leuven, Celestijnenlaan $200 F$, B-3001 Leuven, Belgium.

wim.dehaen@chem.kuleuven.be; Fax: +3216327990; Tel.: +3216327390

Table of contents

1. General experimental methods $\quad$ S2

$\begin{array}{ll}\text { 2. Experimental and characterization data } & \text { S2 }\end{array}$

3. Mass spectrum for the oxacalix[n]arene mixture originally obtained for 3-7a S5

4. ${ }^{1} \mathrm{H}$ NMR spectrum for the oxacalix $[n]$ arene mixture originally obtained for 3-7a $\quad$ S6

5. ${ }^{1} \mathrm{H}$ and ${ }^{13} \mathrm{C}$ NMR spectra for oxacalix[n]arenes 3-7a, 3b-i and thiacalix[4]arene $8 \quad$ S7

6. X-ray crystallographic structure and data for oxacalix[4]arene 3a $\quad$ S19 


\section{General experimental methods}

NMR spectra were acquired on commercial instruments (Bruker Avance $300 \mathrm{MHz}$ or Bruker AMX $400 \mathrm{MHz})$ and chemical shifts $(\delta)$ are reported in parts per million $(\mathrm{ppm})$ referenced to tetramethylsilane (TMS) $\left({ }^{1} \mathrm{H}\right)$ or the carbon signal of deuterated solvents $\left({ }^{13} \mathrm{C}\right)$. Detailed ${ }^{13} \mathrm{C}$ NMR peak assignments were obtained by careful analysis of DEPT, HMQC and HMBC NMR spectra. Mass spectra were run using a HP5989A apparatus (CI and EI, $70 \mathrm{eV}$ ionisation energy) with Apollo 300 data system, a Kratos MS50TC instrument for exact mass measurements (performed in the EI mode at a resolution of 10000), a Micromass Quattro II apparatus (electrospray ionization (ESI), usual solvent mixture: $\mathrm{CH}_{2} \mathrm{Cl}_{2}-\mathrm{MeOH}$ $+\mathrm{NH}_{4} \mathrm{OAc}$ ) with MASSLYNX data system or a Thermo Finnigan LCQ Advantage apparatus (APCI/ESI, solvent $\mathrm{CH}_{3} \mathrm{OH}$ or $\mathrm{CH}_{3} \mathrm{CN}$ ). UV-vis spectra were taken on a Perkin-Elmer Lambda 20 Spectrometer. IR-spectra were recorded on a Perkin-Elmer 1600 Infrared Fourier Transform Spectrometer as KBr pellets. Elemental analysis (C, H and N) of powdered samples was performed on a CE Instrument EA-1110 elemental analyzer. Melting points (not corrected) were determined using a Reichert Thermovar apparatus. For column chromatography 70-230 mesh silica 60 (E.M. Merck) was used as the stationary phase. Chemicals received from commercial sources were used without further purification. DMF was dried on molecular sieves $4 \AA . \mathrm{K}_{2} \mathrm{CO}_{3}$ (anhyd, granulated) was ground very well (with mortar and pestle) prior to use.

\section{Experimental and characterization data}

4,6-Difluoro-2-phenylpyrimidine (1b): This compound has been prepared before by Burdeska et al. (ref. 13). Synthesis according to Plé et al. (ref. 14). Material identity was confirmed by $\mathrm{MS},{ }^{1} \mathrm{H}$ and ${ }^{13} \mathrm{C}$ NMR, IR and $\mathrm{mp}$.

Synthesis of a mixture of oxacalix[4]arene $3 a$ and the larger oxacalix[n]arenes (4-7a): 4,6Dichloro-2-phenylpyrimidine (1a) $(100 \mathrm{mg}, 0.44 \mathrm{mmol})$, orcinol (2a) $(55 \mathrm{mg}, 0.44 \mathrm{mmol})$ and $\mathrm{K}_{2} \mathrm{CO}_{3}$ $(153 \mathrm{mg}, 1.1 \mathrm{mmol})$ were combined in DMF $(5 \mathrm{~mL})$ and this mixture was heated at $70{ }^{\circ} \mathrm{C}$ for $48 \mathrm{~h}$ (under Ar). DMF was removed under vacuum and the mixture was redissolved in ethyl acetate (100 $\mathrm{mL})$ and washed with water $(3 \times 50 \mathrm{~mL})$. The organic fraction was dried over $\mathrm{MgSO}_{4}$, filtered and the solvent was removed under vacuum. After column chromatographic separation (silica, eluent $\mathrm{CH}_{2} \mathrm{Cl}_{2}$ heptane 2-1), oxacalix[4]arene 3a (37 mg, 30\%), oxacalix[6] arene 4a (10 mg, 8\%), oxacalix[8] arene 5a $(12 \mathrm{mg}, 10 \%)$, oxacalix[10]arene 6a $(10 \mathrm{mg}, 8 \%)$ and oxacalix[12]arene $7 \mathbf{a}(10 \mathrm{mg}, 8 \%)$ were isolated in pure form.

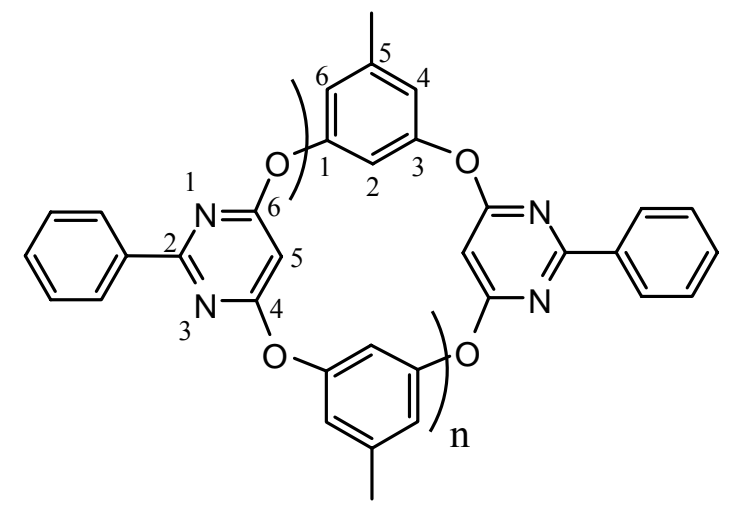

Oxacalix[4]arene 3a (4,6,16,18-tetraaza-11,23-dimethyl-5,17-diphenyl-2,8,14,20-tetraoxacalix[4]arene): $\mathrm{MS}(\mathrm{APCI}+) \mathrm{m} / \mathrm{z} 553.7\left[\mathrm{MH}^{+}\right]$; HRMS (EI) calcd for $\mathrm{C}_{34} \mathrm{H}_{24} \mathrm{~N}_{4} \mathrm{O}_{4}$ : 552.1798; found: $\mathrm{m} / \mathrm{z}$ $552.1792 ;{ }^{1} \mathrm{H}$ NMR $\left(400 \mathrm{MHz}, \mathrm{CDCl}_{3}\right) \delta 8.48\left(\mathrm{dd},{ }^{3} \mathrm{~J}=8.20 \mathrm{~Hz},{ }^{4} \mathrm{~J}=1.84 \mathrm{~Hz}, 4 \mathrm{H}\right.$; o-phenyl), $7.52-7.44$ (m, 6H; m,p-phenyl), $6.93\left(\mathrm{~d},{ }^{4} J=1.84 \mathrm{~Hz}, 4 \mathrm{H} ; 4,6\right.$-orc), 6.68 (s, 2H; 2-orc), 5.36 (s, 2H; pyr), $2.40\left(\mathrm{~s}, 6 \mathrm{H} ; \mathrm{CH}_{3}\right) ;{ }^{13} \mathrm{C}$ NMR $\left(75 \mathrm{MHz}^{\mathrm{CDCl}} \mathrm{CD}_{3}\right) \delta 172.8(\mathrm{C} ; 4,6$-pyr), $166.3(\mathrm{C} ; 2$-pyr), $152.8(\mathrm{C}$; 1,3-orc), 143.7 (C; 5-orc), 135.8 (C; ipso-phenyl), 131.6 (CH; p-phenyl), 128.6 (CH; o/m-phenyl), $128.4\left(\mathrm{CH} ; \mathrm{o} / \mathrm{m}\right.$-phenyl), $120.4\left(\mathrm{CH}\right.$; 4,6-orc), $111.5\left(\mathrm{CH}\right.$; 2-orc), 85.3 (CH; 5-pyr), $21.4\left(\mathrm{CH}_{3}\right)$; UV-vis $\left(\mathrm{CH}_{2} \mathrm{Cl}_{2}\right) \lambda_{\max }(\log \varepsilon) 267.6$ (4.647); IR (KBr) $v_{\max }\left(\mathrm{cm}^{-1}\right)$ 3447, 3059, 2922, 1573, 1555, 1458, 1410, $1357,1304,1165,1117,1035,1002,989,867,821,755,684$; Anal. calcd for $\mathrm{C}_{34} \mathrm{H}_{24} \mathrm{~N}_{4} \mathrm{O}_{4}$ : C, 73.90; $\mathrm{H}$, $4.38 ; \mathrm{N}, 10.14$, found: $\mathrm{C}, 73.55 ; \mathrm{H}, 4.33 ; \mathrm{N}, 9.79 ; \mathrm{mp} 313.0-314.0^{\circ} \mathrm{C}$. 
Oxacalix[6]arene 4a $(4,6,16,18,28,30$-hexaaza-11,23,35-trimethyl-5,17,29-triphenyl-2,8,14,20,26, 32-hexaoxacalix[6]arene): In order to obtain oxacalix[6]arene 4a in pure form, a second chromatographic purification had to be performed (silica, eluent toluene-ethyl acetate 99-1). After this, the product still contained a small amount $(<10 \%)$ of oxacalix[10]arene 6a. MS (APCI +$)$ calcd for $\mathrm{C}_{51} \mathrm{H}_{36} \mathrm{~N}_{6} \mathrm{O}_{6}$ : 828.9; found: $\mathrm{m} / \mathrm{z} 829.6\left[\mathrm{MH}^{+}\right] ;{ }^{1} \mathrm{H}$ NMR $\left(300 \mathrm{MHz}, \mathrm{CDCl}_{3}\right) \delta 8.24\left(\mathrm{~d},{ }^{3} \mathrm{~J}=8.22 \mathrm{~Hz}, 6 \mathrm{H}\right.$; o-phenyl), 7.46-7.33 (m, 9H; m,p-phenyl), 6.95 (s, 6H; 4,6-orc), 6.69 (s, 3H; 2-orc), 5.95 (s, 3H; pyr), $2.44\left(\mathrm{~s}, 9 \mathrm{H} ; \mathrm{CH}_{3}\right) ;{ }^{13} \mathrm{C}$ NMR $\left(75 \mathrm{MHz}, \mathrm{CDCl}_{3}\right) \delta 172.1$ (C; 4,6-pyr), 165.4 (C; 2-pyr), $153.2(\mathrm{C} ; 1,3-$ orc), 142.2 (C; 5-orc), 135.9 (C; ipso-phenyl), 131.5 (CH; p-phenyl), 128.5 (CH; o,m-phenyl), 119.4 (CH; 4,6-orc), 111.2 (CH; 2-orc), $88.8\left(\mathrm{CH} ; 5\right.$-pyr), $21.5\left(\mathrm{CH}_{3}\right)$.

Oxacalix[8]arene $5 a \quad(4,6,16,18,28,30,40,42$-octaaza-11,23,35,47-tetramethyl-5,17,29,41-tetraphenyl-2,8,14,20,26,32,38,44-octaoxacalix[8]arene): $M S$ (APCI+) calcd for $\mathrm{C}_{68} \mathrm{H}_{48} \mathrm{~N}_{8} \mathrm{O}_{8}$ : 1105.2 ; found: $\mathrm{m} / \mathrm{z} 1105.6\left[\mathrm{MH}^{+}\right] ;{ }^{1} \mathrm{H}$ NMR $\left(300 \mathrm{MHz}, \mathrm{CDCl}_{3}\right) \delta 8.07\left(\mathrm{~d},{ }^{3} \mathrm{~J}=7.29 \mathrm{~Hz}, 8 \mathrm{H} ; o\right.$-phenyl), $7.21(\mathrm{t}$, ${ }^{3} J=7.29 \mathrm{~Hz}, 4 \mathrm{H}$; p-phenyl), $7.11\left(\mathrm{t},{ }^{3} \mathrm{~J}=7.29 \mathrm{~Hz}, 8 \mathrm{H} ; \mathrm{m}\right.$-phenyl), 6.98 (s, 8H; 4,6-orc), 6.76 (s, 4H; 2orc), 6.16 (s, 4H; pyr), $2.47\left(\mathrm{~s}, 12 \mathrm{H} ; \mathrm{CH}_{3}\right) ;{ }^{13} \mathrm{C}$ NMR (100 MHz, $\left.\mathrm{CDCl}_{3}\right) \delta 171.6(\mathrm{C} ; 4,6-\mathrm{pyr}), 164.2(\mathrm{C}$; 2-pyr), 153.1 (C; 1,3-orc), 141.1 (C; 5-orc), 136.1 (C; ipso-phenyl), 131.1 (CH; p-phenyl), $128.3(\mathrm{CH}$; o,m-phenyl), 119.5 (CH; 4,6-orc), 112.3 (CH; 2-orc), $89.9\left(\mathrm{CH} ; 5\right.$-pyr), $21.5\left(\mathrm{CH}_{3}\right) ; \mathrm{mp} 254.7-255.7$ ${ }^{\circ} \mathrm{C}$.

Oxacalix[10]arene 6a $(4,6,16,18,28,30,40,42,52,54$-decaaza-11,23,35,47,59-pentamethyl-5,17,29, 41,53-pentaphenyl-2,8,14,20,26,32,38,44,50,56-decaoxacalix[10]arene): $\mathrm{MS}$ (APCI + ) calcd for $\mathrm{C}_{85} \mathrm{H}_{60} \mathrm{~N}_{10} \mathrm{O}_{10}$ : 1381.4; found: $\mathrm{m} / \mathrm{z} 1382.4\left[\mathrm{MH}^{+}\right]$; ${ }^{1} \mathrm{H}$ NMR $\left(300 \mathrm{MHz}, \mathrm{CDCl}_{3}\right) \delta 8.12\left(\mathrm{~d},{ }^{3} \mathrm{~J}=7.29 \mathrm{~Hz}\right.$, $10 \mathrm{H}$; o-phenyl), $7.24\left(\mathrm{t},{ }^{3} \mathrm{~J}=7.29 \mathrm{~Hz}, 5 \mathrm{H}\right.$; p-phenyl), $7.16\left(\mathrm{t},{ }^{3} \mathrm{~J}=7.29 \mathrm{~Hz}, 10 \mathrm{H} ; \mathrm{m}\right.$-phenyl), $6.95(\mathrm{~s}$, $10 \mathrm{H} ; 4,6$-orc), 6.86 (s, 5H; 2-orc), 6.17 (s, 5H; pyr), $2.43\left(\mathrm{~s}, 15 \mathrm{H} ; \mathrm{CH}_{3}\right) ;{ }^{13} \mathrm{C} \mathrm{NMR}\left(75 \mathrm{MHz}, \mathrm{CDCl}_{3}\right) \delta$ 171.7 (C; 4,6-pyr), 164.4 (C; 2-pyr), 153.2 (C; 1,3-orc), 141.0 (C; 5-orc), 136.2 (C; ipso-phenyl), 131.2 (CH; p-phenyl), 128.3 (CH; o,m-phenyl), 119.4 (CH; 4,6-orc), 112.6 (CH; 2-orc), 89.6 (CH; 5-pyr), $21.5\left(\mathrm{CH}_{3}\right)$.

Oxacalix[12]arene $\quad 7$ a $\quad(4,6,16,18,28,30,40,42,52,54,64,66$-dodecaaza-11,23,35,47,59,71-hexamethyl-5,17,29,41,53,65-hexaphenyl-2,8,14,20,26,32,38,44,50,56,62,68-dodecaoxacalix[12]arene): MS (APCI+) calcd for $\mathrm{C}_{102} \mathrm{H}_{72} \mathrm{~N}_{12} \mathrm{O}_{12}$ : 1657.7; found: $\mathrm{m} / \mathrm{z} 1658.6\left[\mathrm{MH}^{+}\right]$; ${ }^{1} \mathrm{H}$ NMR $\left(300 \mathrm{MHz}, \mathrm{CDCl}_{3}\right.$ ) $\delta 8.14\left(\mathrm{dd},{ }^{3} \mathrm{~J}=7.29 \mathrm{~Hz},{ }^{4} \mathrm{~J}=1.83 \mathrm{~Hz}, 12 \mathrm{H}\right.$; o-phenyl), $7.26\left(\mathrm{t},{ }^{3} \mathrm{~J}=7.32 \mathrm{~Hz}, 6 \mathrm{H} ; p\right.$-phenyl), $7.18\left(\mathrm{t},{ }^{3} \mathrm{~J}\right.$ $=7.32 \mathrm{~Hz}, 12 \mathrm{H} ;$ m-phenyl), 6.93 (d, ${ }^{4} \mathrm{~J}=1.83 \mathrm{~Hz}, 12 \mathrm{H} ; 4,6$-orc), 6.88 (s, 6H; 2-orc), 6.15 (s, $6 \mathrm{H}$; pyr), 2.41 (s, 18H; $\left.\mathrm{CH}_{3}\right) ;{ }^{13} \mathrm{C}$ NMR (75 MHz, $\left.\mathrm{CDCl}_{3}\right) \delta 171.7$ (C; 4,6-pyr), 164.4 (C; 2-pyr), $153.2(\mathrm{C} ; 1,3-$ orc), 141.0 (C; 5-orc), 136.2 (C; ipso-phenyl), 131.2 (CH; p-phenyl), 128.3 (CH; o,m-phenyl), 119.4 (CH; 4,6-orc), 112.6 (CH; 2-orc), $89.6\left(\mathrm{CH} ; 5\right.$-pyr), $21.5\left(\mathrm{CH}_{3}\right)$.

Optimized synthesis of oxacalix[4]arene 3a (general thia- and oxacalix[4]arene synthesis): 4,6Difluoro-2-phenylpyrimidine (1b) (100 mg, $0.52 \mathrm{mmol})$, orcinol (2a) $(65 \mathrm{mg}, 0.52 \mathrm{mmol}), \mathrm{K}_{2} \mathrm{CO}_{3}(180$ $\mathrm{mg}, 1.30 \mathrm{mmol})$ and $18 \mathrm{C} 6(22 \mathrm{mg}, 83 \mu \mathrm{mol})$ were combined in DMF $(5 \mathrm{~mL})$ and the mixture was heated at $70{ }^{\circ} \mathrm{C}$ for $24 \mathrm{~h}$ (under Ar). DMF was removed under vacuum and the mixture was redissolved in $\mathrm{CH}_{2} \mathrm{Cl}_{2}(100 \mathrm{~mL})$ and washed with water $(3 \times 50 \mathrm{~mL})$. The organic fraction was dried over $\mathrm{MgSO}_{4}$, filtered and the solvent was removed under vacuum. Oxacalix[4]arene 3a was purified by column chromatography (silica, eluent $\mathrm{CH}_{2} \mathrm{Cl}_{2}$-heptane 3-1) and obtained as a white solid (112 $\mathrm{mg}, 78 \%$ ).

Oxacalix[4]arene 3b (4,6,16,18-tetraaza-11,23-dimethyl-2,8,14,20-tetraoxacalix[4]arene): 4,6Dichloropyrimidine (1c) (200 mg, $1.34 \mathrm{mmol}$ ), orcinol (2a) (167 mg, $1.34 \mathrm{mmol}), \mathrm{K}_{2} \mathrm{CO}_{3}(464 \mathrm{mg}, 3.36$ mmol), $18 \mathrm{C} 6$ (77 mg, $0.29 \mathrm{mmol}$ ), $48 \mathrm{~h}$; Eluent $\mathrm{CH}_{2} \mathrm{Cl}_{2}$-ethyl acetate 2-1; Yield 75\% (202 mg); MS (ESI+) $\mathrm{m} / \mathrm{z} 401.3\left[\mathrm{MH}^{+}\right]$; HRMS (EI) calcd for $\mathrm{C}_{22} \mathrm{H}_{16} \mathrm{~N}_{4} \mathrm{O}_{4}$ : 400.1172; found: $\mathrm{m} / \mathrm{z}$ 400.1179; ${ }^{1} \mathrm{H}$ NMR $\left(300 \mathrm{MHz}, \mathrm{CDCl}_{3}\right) \delta 8.66$ (s, 2H; 2-pyr), 6.94 (d, ${ }_{4} J=1.83 \mathrm{~Hz}, 4 \mathrm{H} ; 4,6$-orc), 6.67 (s, 2H; 2-orc), 5.46 (s, 2H; 5-pyr), 2.40 (s, 6H; $\left.\mathrm{CH}_{3}\right) ;{ }^{13} \mathrm{C}$ NMR (75 MHz, $\left.\mathrm{CDCl}_{3}\right) \delta 172.5$ (C; 4,6-pyr), 159.9 (CH; 2-pyr), 152.6 (C; 1,3-orc), 144.0 (C; 5-orc), 120.6 (CH; 4,6-orc), 111.2 (CH; 2-orc), 87.5 (CH; 5-pyr), 21.3 $\left(\mathrm{CH}_{3}\right)$; IR $(\mathrm{KBr}) v_{\max }\left(\mathrm{cm}^{-1}\right) 3055,2922,1574,1463,1373,1295,1236,1171,1130,1023,992,872$, 826, 765, 731, 682; Anal. calcd for $\mathrm{C}_{22} \mathrm{H}_{16} \mathrm{~N}_{4} \mathrm{O}_{4}$ : C, 66.00; $\mathrm{H}, 4.03$; N, 13.99, found: $\mathrm{C}, 65.58$; $\mathrm{H}, 4.06$; $\mathrm{N}, 13.74 ; \mathrm{mp} 278.4-279.4{ }^{\circ} \mathrm{C}$.

Oxacalix[4]arene 3c (4,6,16,18-tetraaza-11,23-dimethyl-5,17-di(thiomethyl)-2,8,14,20-tetraoxacalix[4]arene): 4,6-Dichloro-2-thiomethylpyrimidine (1d) $(200 \mathrm{mg}, 1.03 \mathrm{mmol})$, orcinol (2a) (127 mg, $1.03 \mathrm{mmol}$ ), $\mathrm{K}_{2} \mathrm{CO}_{3}(354 \mathrm{mg}, 2.6 \mathrm{mmol}), 18 \mathrm{C} 6$ (58 mg, $0.22 \mathrm{mmol}$ ), $48 \mathrm{~h}$; Eluent $\mathrm{CH}_{2} \mathrm{Cl}_{2}$-heptane 3-1; 
Yield 65\% (164 mg); MS (APCI+) m/z 493.4 [MH ${ }^{+}$; HRMS (EI) calcd for $\mathrm{C}_{24} \mathrm{H}_{20} \mathrm{~N}_{4} \mathrm{O}_{4} \mathrm{~S}_{2}$ : 492.0926 ; found: $\mathrm{m} / \mathrm{z}$ 492.0931; ${ }^{1} \mathrm{H} \mathrm{NMR}\left(300 \mathrm{MHz}, \mathrm{CDCl}_{3}\right) \delta 6.88$ (d, ${ }^{4} \mathrm{~J}=1.83 \mathrm{~Hz}, 4 \mathrm{H} ; 4,6$-orc), 6.59 (s, 2H; 2orc), 5.03 (s, 2H; 5-pyr), $2.60\left(\mathrm{~s}, 6 \mathrm{H} ; \mathrm{S}-\mathrm{CH}_{3}\right), 2.37\left(\mathrm{~s}, 6 \mathrm{H} ; \mathrm{CH}_{3}\right) ;{ }^{13} \mathrm{C} \mathrm{NMR}\left(75 \mathrm{MHz}, \mathrm{CDCl}_{3}\right) \delta 175.0$ (C; 2-pyr), 172.0 (C; 4,6-pyr), 152.7 (C; 1,3-orc), 143.7 (C; 5-orc), 120.5 (CH; 4,6-orc), 111.6 (CH; 2orc), 82.8 (CH; 5-pyr), $21.5\left(\mathrm{CH}_{3}\right.$; methyl), $14.2\left(\mathrm{CH}_{3}\right.$; S-methyl); IR (KBr) v $\max \left(\mathrm{cm}^{-1}\right) 3058,2927$, $1558,1462,1410,1367,1285,1168,1135,1038,998,910,865,808,727,680$; Anal. calcd for $\mathrm{C}_{24} \mathrm{H}_{20} \mathrm{~N}_{4} \mathrm{O}_{4} \mathrm{~S}_{2}$ : C, 58.52; H, 4.09; N, 11.37, found: C, 58.61; H, 4.10; N, 11.07; mp 267.1-268.1 ${ }^{\circ} \mathrm{C}$.

Oxacalix[4]arene 3d (4,6,16,18-tetraaza-11,23-dimethyl-5-thiomethyl-2,8,14,20-tetraoxacalix[4]arene): 4,6-Dichloropyrimidine (1c) $(109 \mathrm{mg}, 0.73 \mathrm{mmol})$, 4,6-dichloro-2-thiomethylpyrimidine (1d) $(143 \mathrm{mg}, 0.73 \mathrm{mmol})$, orcinol (2a) $(181 \mathrm{mg}, 1.46 \mathrm{mmol}), \mathrm{K}_{2} \mathrm{CO}_{3}(464 \mathrm{mg}, 3.36 \mathrm{mmol}), 18 \mathrm{C} 6$ (60 $\mathrm{mg}, 0.23 \mathrm{mmol}), 48 \mathrm{~h}$; Eluent $\mathrm{CH}_{2} \mathrm{Cl}_{2}$-ethyl acetate 2-1; Yield 43\% (139 mg) 3d, 30\% (107 mg) 3c, $18 \%$ (54 mg) 3b; MS (APCI+) m/z 447.4 [MH ${ }^{+}$; HRMS (EI) calcd for $\mathrm{C}_{23} \mathrm{H}_{18} \mathrm{~N}_{4} \mathrm{O}_{4} \mathrm{~S}$ : 446.1049; found: m/z 446.1050; ${ }^{1} \mathrm{H}$ NMR (300 MHz, $\mathrm{CDCl}_{3}$ ) $\delta 8.65$ (s, 1H; 2-pyr), 6.92 (s, 4H; 4,6-orc), 6.66 (s, 2H; 2orc), $5.44(\mathrm{~s}, 1 \mathrm{H} ; 5-\mathrm{pyr}-\mathrm{H}), 5.06\left(\mathrm{~s}, 1 \mathrm{H} ; 5-\mathrm{pyr}_{-} \mathrm{SCH}_{3}\right), 2.58\left(\mathrm{~s}, 3 \mathrm{H} ; \mathrm{S}_{-} \mathrm{CH}_{3}\right), 2.39\left(\mathrm{~s}, 6 \mathrm{H} ; \mathrm{CH}_{3}\right) ;{ }^{3} \mathrm{C} \mathrm{NMR}$ $\left.\left(75 \mathrm{MHz}, \mathrm{CDCl}_{3}\right) \delta 174.6(\mathrm{C} ; 2 \text {-pyr-SCH})_{3}\right), 172.3$ and 171.8 (C; 4,6-pyr), $159.7(\mathrm{CH} ; 2$-pyr-H), 152.5 and $152.3(\mathrm{C}$; 1,3-orc), 143.7 (C; 5-orc), 120.4 and $120.3(\mathrm{CH} ; 4,6$-orc), $111.2(\mathrm{CH}$; 2-orc), $87.4(\mathrm{CH}$; 5-pyr-H), 82.6 (CH; 5-pyr-SCH 3$), 21.4\left(\mathrm{CH}_{3}\right.$; methyl), $14.2\left(\mathrm{CH}_{3}\right.$; S-methyl); IR $(\mathrm{KBr}) v_{\max }\left(\mathrm{cm}^{-1}\right)$ 3056, 2927, 1572, 1460, 1369, 1295, 1169, 1132, 1032, 994, 860, 826, 804, 681; Anal. calcd for $\mathrm{C}_{23} \mathrm{H}_{18} \mathrm{~N}_{4} \mathrm{O}_{4} \mathrm{~S} . \mathrm{H}_{2} \mathrm{O}: \mathrm{C}, 59.47 ; \mathrm{H}, 4.34 ; \mathrm{N}, 12.06$, found: $\mathrm{C}, 59.19 ; \mathrm{H}, 3.88 ; \mathrm{N}, 11.73 ; \mathrm{mp} 260.8-261.8$ ${ }^{\circ} \mathrm{C}$.

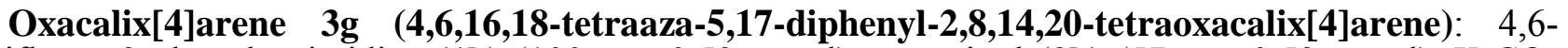
Difluoro-2-phenylpyrimidine (1b) $(100 \mathrm{mg}, 0.52 \mathrm{mmol})$, resorcinol (2b) $(57 \mathrm{mg}, 0.52 \mathrm{mmol}), \mathrm{K}_{2} \mathrm{CO}_{3}$ $(180 \mathrm{mg}, 1.30 \mathrm{mmol}), 18 \mathrm{C} 6$ (26 mg, $0.10 \mathrm{mmol}), 24 \mathrm{~h}$; Eluent $\mathrm{CH}_{2} \mathrm{Cl}_{2}$; Yield 80\% (109 mg); MS (APCI+) $\mathrm{m} / \mathrm{z} 525.4\left[\mathrm{MH}^{+}\right]$; HRMS (EI) calcd for $\mathrm{C}_{32} \mathrm{H}_{20} \mathrm{~N}_{4} \mathrm{O}_{4}$ : 524.1485; found: $\mathrm{m} / \mathrm{z} 524.1483$; ${ }^{1} \mathrm{H}$ NMR $\left(300 \mathrm{MHz}, \mathrm{CDCl}_{3}\right) \delta 8.49\left(\mathrm{dd},{ }^{3} \mathrm{~J}=7.32 \mathrm{~Hz},{ }^{4} \mathrm{~J}=1.83 \mathrm{~Hz}, 4 \mathrm{H} ; o\right.$-phenyl), 7.56-7.45 (m, 8H; $m, p$ phenyl, 5-resorc), 7.14 ( $\left(\mathrm{dd}^{3} \mathrm{~J}=8.22 \mathrm{~Hz},{ }^{4} \mathrm{~J}=2.76 \mathrm{~Hz}, 4 \mathrm{H} ; 4,6\right.$-resorc), 6.90 (t, ${ }^{4} \mathrm{~J}=1.83 \mathrm{~Hz}, 2 \mathrm{H}$; 2 resorc), 5.24 (s, 2H; pyr); ${ }^{13} \mathrm{C}$ NMR (75 MHz, $\left.\mathrm{CDCl}_{3}\right) \delta 173.0$ (C; 4,6-pyr), 166.5 (C; 2-pyr), $153.2(\mathrm{C}$; 1,3-resorc), 135.7 (C; ipso-phenyl), $132.8(\mathrm{CH} ; 5$-resorc),131.7 (CH; p-phenyl), $128.7(\mathrm{CH} ;$ o/mphenyl), 128.5 (CH; o/m-phenyl), $120.1(\mathrm{CH} ; 4,6$-resorc), $115.0(\mathrm{CH} ; 2$-resorc), $85.2(\mathrm{CH}$; 5-pyr); IR $(\mathrm{KBr}) v_{\max }\left(\mathrm{cm}^{-1}\right) 3065,1584,1554,1481,1408,1361,1266,1232,1160,1119,1024,985,816,752$, 694; Anal. calcd for $\mathrm{C}_{32} \mathrm{H}_{20} \mathrm{~N}_{4} \mathrm{O}_{4}$ : C, 73.27; H, 3.84; N, 10.68, found: C, 72.83; H, 3.91; N, 10.46; mp > $300^{\circ} \mathrm{C}$.

Oxacalix[4]arene $3 \mathrm{~h}$ (4,6,16,18-tetraaza-26,28-dihydroxy-5,17-diphenyl-2,8,14,20-tetraoxacalix[4]arene): 4,6-Difluoro-2-phenylpyrimidine (1b) $(100 \mathrm{mg}, 0.52 \mathrm{mmol})$, pyrogallol (2c) $(66 \mathrm{mg}, 0.52$ $\mathrm{mmol}), \mathrm{K}_{2} \mathrm{CO}_{3}(180 \mathrm{mg}, 1.30 \mathrm{mmol}), 18 \mathrm{C} 6(26 \mathrm{mg}, 0.10 \mathrm{mmol}), 24 \mathrm{~h}$, use of $0.1 \mathrm{~N} \mathrm{HCl}$ for initial washing; Eluent ethyl acetate-petroleum ether 3-7; Yield 31\% (45 mg); MS (APCI+) m/z 557.2 [MH ${ }^{+}$; HRMS (EI) calcd for $\mathrm{C}_{32} \mathrm{H}_{20} \mathrm{~N}_{4} \mathrm{O}_{6}: 556.1383$; found: $\mathrm{m} / \mathrm{z} 556.1391 ;{ }^{1} \mathrm{H}$ NMR (300 MHz, DMSO- $d_{6^{-}}$ $\left.\mathrm{CDCl}_{3} 1-1\right) \delta 10.17(\mathrm{~s}, 2 \mathrm{H} ; \mathrm{OH}), 8.47\left(\mathrm{dd},{ }^{3} \mathrm{~J}=6.86 \mathrm{~Hz},{ }^{4} \mathrm{~J}=2.27 \mathrm{~Hz}, 4 \mathrm{H} ; o\right.$-phenyl), 7.54-7.50 (m, 6H; m,p-phenyl), 7.07 (d, ${ }^{3} J=8.22 \mathrm{~Hz}, 4 \mathrm{H} ; 4,6-$ pyrog), 6.91 (t, ${ }^{3} J=8.22 \mathrm{~Hz}, 2 \mathrm{H} ; 5$-pyrog), 5.03 (s, 2H; pyr); ${ }^{13} \mathrm{C}$ NMR (75 MHz, DMSO-d $\left.{ }_{6}-\mathrm{CDCl}_{3} 1-1\right) \delta 171.9$ (C; 4,6-pyr), 163.9 (C; 2-pyr), 141.9 (C; 2pyrog), 140.8 (C; 1,3-pyrog), 135.8 (C; ipso-phenyl), 130.9 (CH; p-phenyl), 128.1 (CH; o/m-phenyl), $127.8(\mathrm{CH}$; o/m-phenyl), $120.7(\mathrm{CH} ; 4,6-$ pyrog), 119.9 (CH; 5-pyrog), 84.1 (CH; 5-pyr) (relative to DMSO-d 6 : 39.5); IR (KBr) $v_{\max }\left(\mathrm{cm}^{-1}\right) 3488,3066,1692,1561,1498,1473,1366,1289,1226,1155$, $1016,818,752,724,697 ; \mathrm{mp} 308.5-310.0{ }^{\circ} \mathrm{C}$.

Oxacalix[4]arene $3 i$ (4,6,16,18-tetraaza-26,28-dimethyl-2,8,14,20-tetraoxacalix[4]arene): 4,6Dichloropyrimidine (1c) $(100 \mathrm{mg}, 0.67 \mathrm{mmol}), 2$-methylresorcinol (2d) (90\% purity; $93 \mathrm{mg}, 0.75$ $\mathrm{mmol}), \mathrm{K}_{2} \mathrm{CO}_{3}(230 \mathrm{mg}, 1.66 \mathrm{mmol}), 18 \mathrm{C} 6(30 \mathrm{mg}, 0.11 \mathrm{mmol}), 24 \mathrm{~h}$; Eluent $\mathrm{CH}_{2} \mathrm{Cl}_{2}$-ethyl acetate 2-1; Yield 15\% (20 mg); MS (APCI+) m/z 401.4 [MH ${ }^{+}$; HRMS (EI) calcd for $\mathrm{C}_{22} \mathrm{H}_{16} \mathrm{~N}_{4} \mathrm{O}_{4}$ : 400.1172 ; found: $\mathrm{m} / \mathrm{z}$ 400.1176; ${ }^{1} \mathrm{H}$ NMR $\left(300 \mathrm{MHz}, \mathrm{CDCl}_{3}\right) \delta 8.73$ (s, 2H; 2-pyr), $7.32\left(\mathrm{t},{ }^{3} \mathrm{~J}=8.22 \mathrm{~Hz}, 2 \mathrm{H} ; 5-\right.$ resorc), $7.04\left(\mathrm{~d},{ }^{3} \mathrm{~J}=8.22 \mathrm{~Hz}, 4 \mathrm{H} ; 4,6\right.$-resorc), $4.74\left(\mathrm{~s}, 2 \mathrm{H} ; 5-\right.$-pyr), $1.79\left(\mathrm{~s}, 6 \mathrm{H} ; \mathrm{CH}_{3}\right) ;{ }^{13} \mathrm{C}$ NMR $(75$ $\left.\mathrm{MHz}, \mathrm{CDCl}_{3}\right) \delta 172.3(\mathrm{C} ; 4,6$-pyr), $160.3(\mathrm{CH} ; 2$-pyr), 151.4 (C; 1,3-resorc), 129.5 (CH; 5-resorc), 124.1 (C, 2-resorc), 120.7 (CH; 4,6-resorc), $86.0\left(\mathrm{CH} ; 5\right.$-pyr), 9.6 $\left(\mathrm{CH}_{3}\right)$; IR $(\mathrm{KBr}) v_{\max }\left(\mathrm{cm}^{-1}\right) 2924$, $1572,1465,1369,1284,1235,1164,1069,1006,829 ; \mathrm{mp}>340^{\circ} \mathrm{C}$. 
Thiacalix[4]arene 8 (4,6,16,18-tetraaza-5,17-diphenyl-2,8,14,20-tetrathiacalix[4]arene): 4,6Difluoro-2-phenylpyrimidine (1b) $(100 \mathrm{mg}, 0.52 \mathrm{mmol}), 1,3$-benzenedithiol (2e) $(60 \mu \mathrm{L}, 0.52 \mathrm{mmol})$, $\mathrm{K}_{2} \mathrm{CO}_{3}$ (180 mg, $\left.1.30 \mathrm{mmol}\right), 18 \mathrm{C} 6(30 \mathrm{mg}, 0.11 \mathrm{mmol}), 48 \mathrm{~h}$; Eluent $\mathrm{CH}_{2} \mathrm{Cl}_{2}$-heptane 3-1; Yield $69 \%$ $\left(105 \mathrm{mg}\right.$ ); MS (APCI+) m/z 589.2 [MH']; HRMS (EI) calcd for $\mathrm{C}_{32} \mathrm{H}_{20} \mathrm{~N}_{4} \mathrm{~S}_{4}$ : 588.0571; found: $\mathrm{m} / \mathrm{z}$ 588.0558; ${ }^{1} \mathrm{H}$ NMR (400 MHz, CDCl $\left.{ }_{3}\right) \delta 8.45\left(\mathrm{dd}^{3} \mathrm{~J}=7.96 \mathrm{~Hz},{ }^{4} \mathrm{~J}=1.82 \mathrm{~Hz}, 4 \mathrm{H} ; \mathrm{o}\right.$-phenyl), $7.79\left(\mathrm{t},{ }^{4} \mathrm{~J}\right.$ $=1.36 \mathrm{~Hz}, 2 \mathrm{H} ; 2$-dithiol), $7.60\left(\mathrm{dd},{ }^{3} \mathrm{~J}=7.72 \mathrm{~Hz},{ }^{4} \mathrm{~J}=1.36 \mathrm{~Hz}, 4 \mathrm{H} ; 4,6\right.$-dithiol), 7.52-7.42 (m, 8H; m, $p$ phenyl, 5-dithiol), 5.64 (s, 2H; pyr); ${ }^{13} \mathrm{C}$ NMR (75 MHz, $\left.\mathrm{CDCl}_{3}\right) \delta 173.1$ (C; 4,6-pyr), 162.9 (C; 2-pyr), 143.6 (CH; 2-dithiol), 138.3 (CH; 4,6-dithiol), 136.1 (C; ipso-phenyl), 131.3 (CH; p-phenyl), 131.1 $(\mathrm{CH}$; 5-dithiol), 130.2 (C; 1,3-dithiol), $128.8(\mathrm{CH}$; o/m-phenyl), $128.5(\mathrm{CH}$; o/m-phenyl), $108.5(\mathrm{CH}$; 5pyr); IR (KBr) $v_{\max }\left(\mathrm{cm}^{-1}\right) 3057,1515,1367,1242,1172,1105,812,747,691,654$; Anal. calcd for $\mathrm{C}_{32} \mathrm{H}_{20} \mathrm{~N}_{4} \mathrm{~S}_{4} \cdot \mathrm{H}_{2} \mathrm{O}$ : C, 63.34; H, 3.65; N, 9.23, found: C, 63.40; H, 3.52; N, 9.09; mp 308.3-309.3 ${ }^{\circ} \mathrm{C}$.

\section{Mass spectrum (APCI+) for the oxacalix[n]arene mixture originally obtained for $3-7$ a}

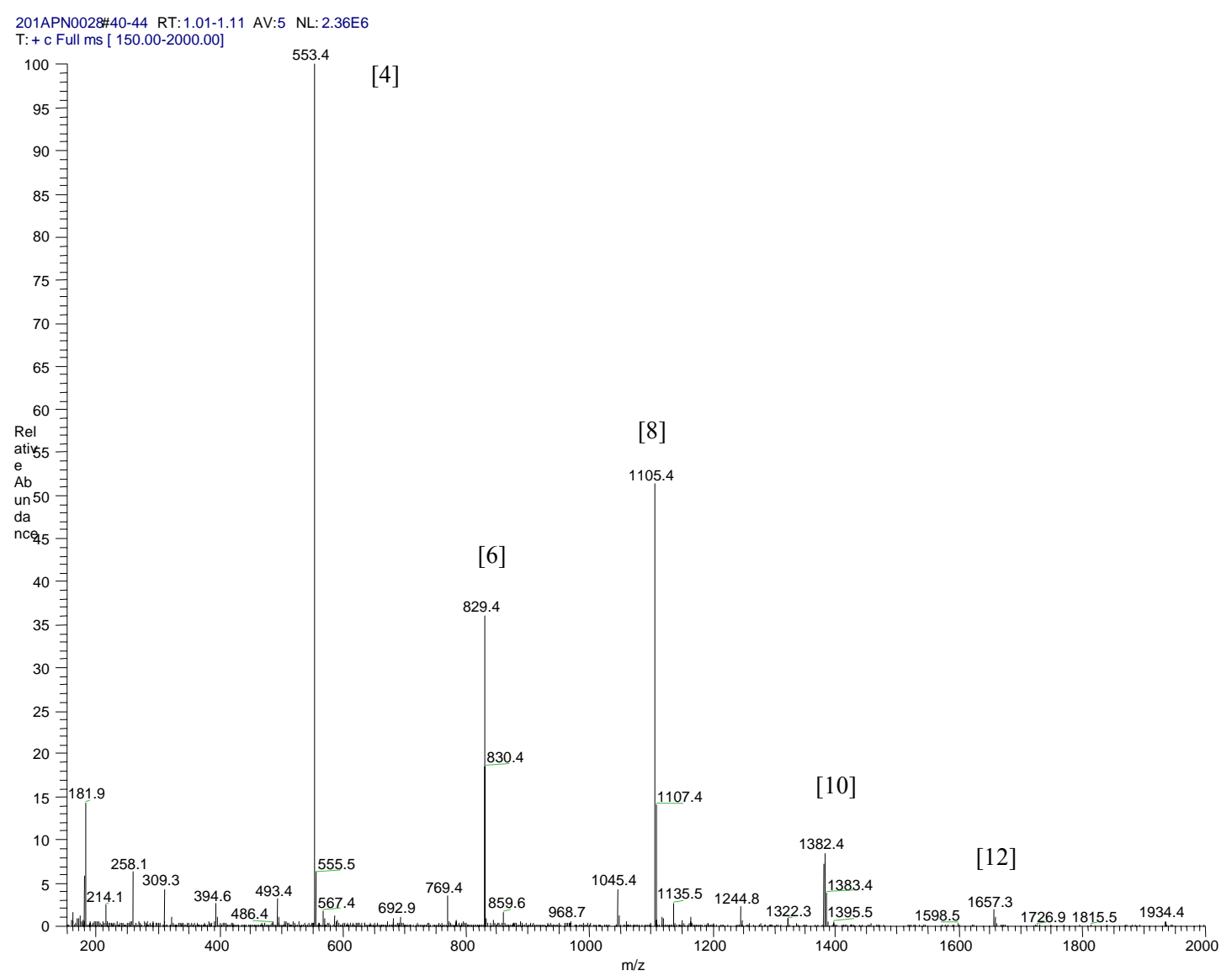


4. ${ }^{1} \mathrm{H}$ NMR spectrum (aromatic region) for the oxacalix[n]arene mixture originally obtained for 3 $7 a$

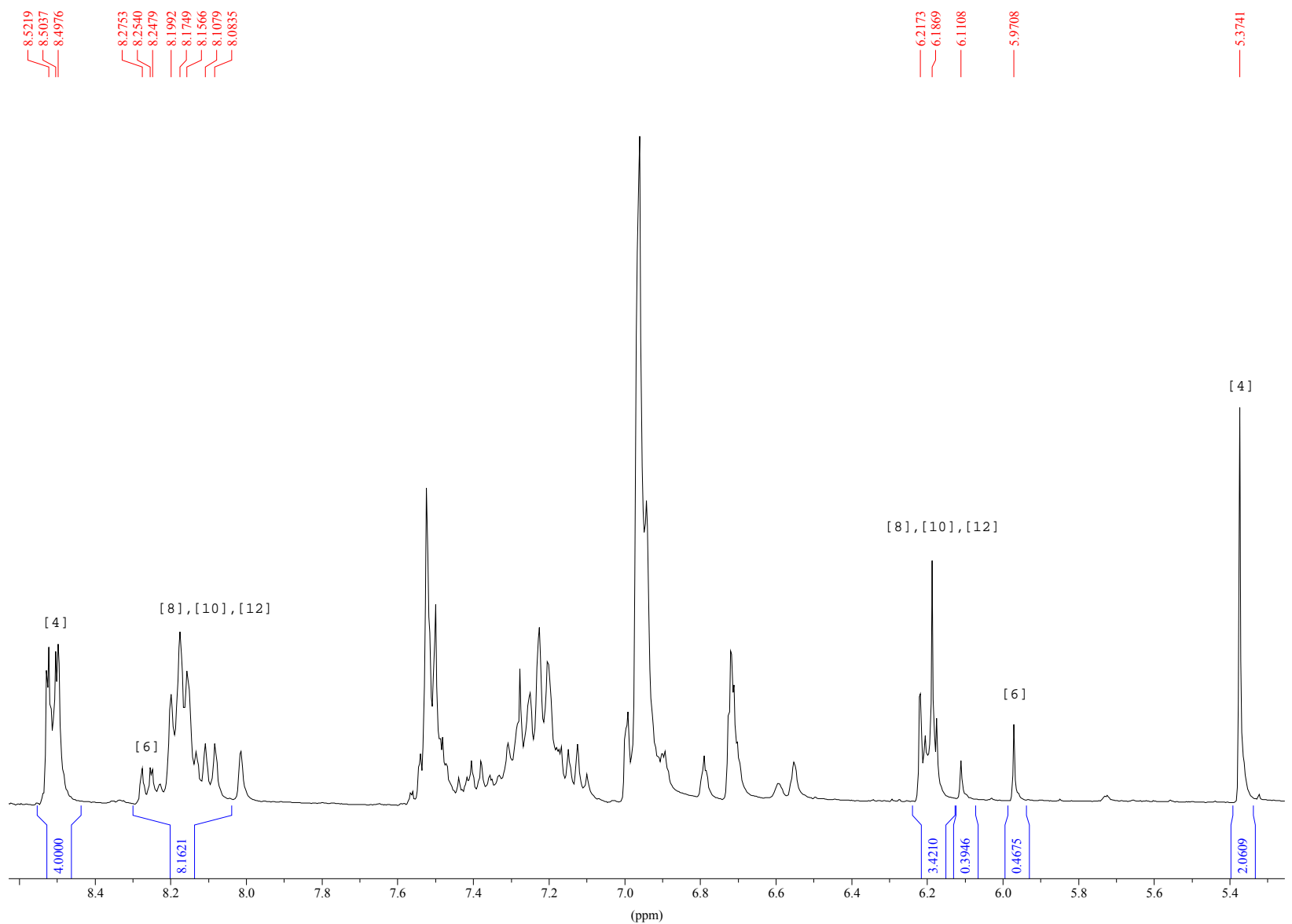


5. ${ }^{1} \mathrm{H}$ and ${ }^{13} \mathrm{C}$ NMR spectra for oxacalix[n]arenes $3-7 \mathrm{a}$, 3b-i and thiacalix[4]arene 8

* Oxacalix[4]arene 3a
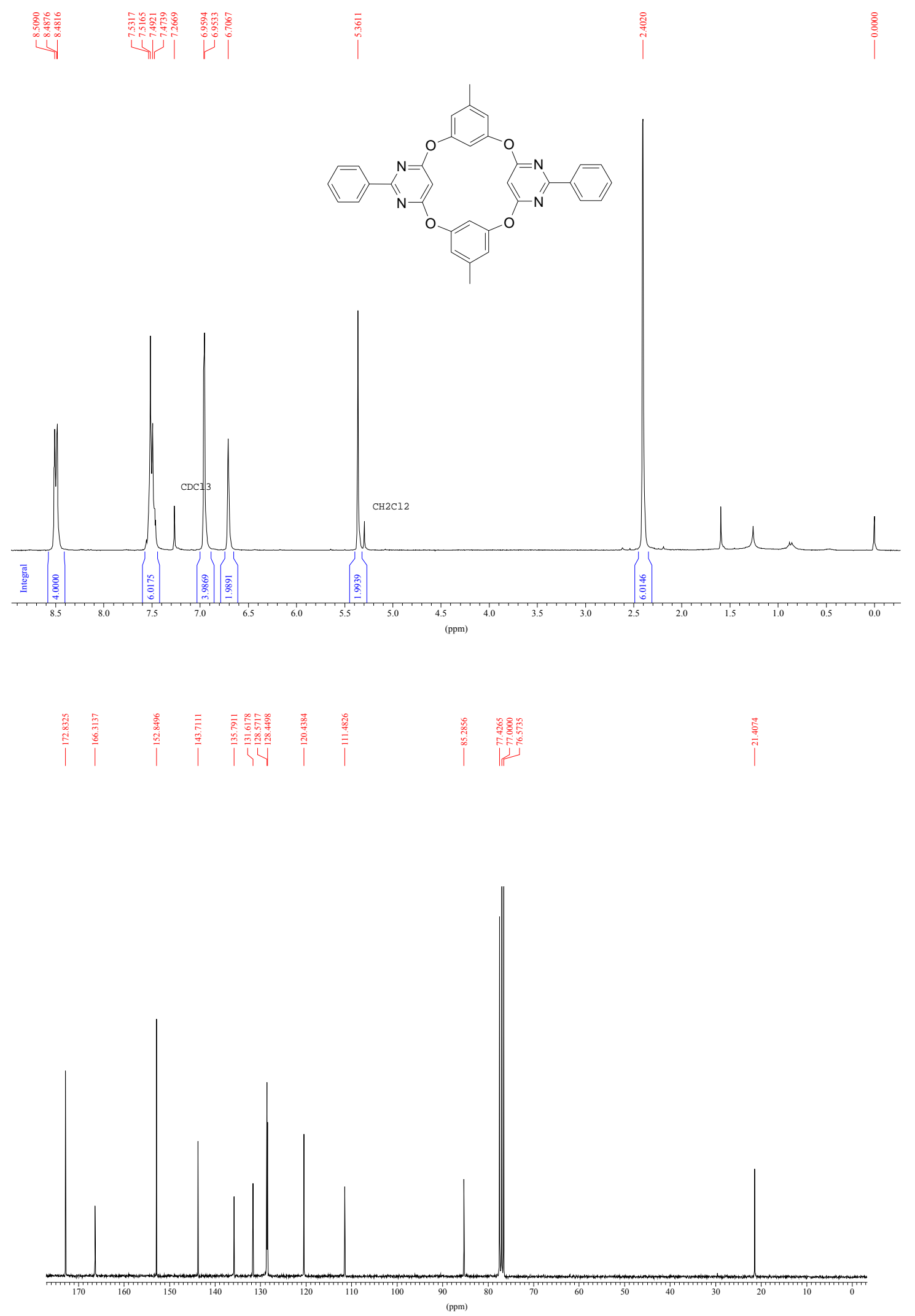
* Oxacalix[6]arene 4a
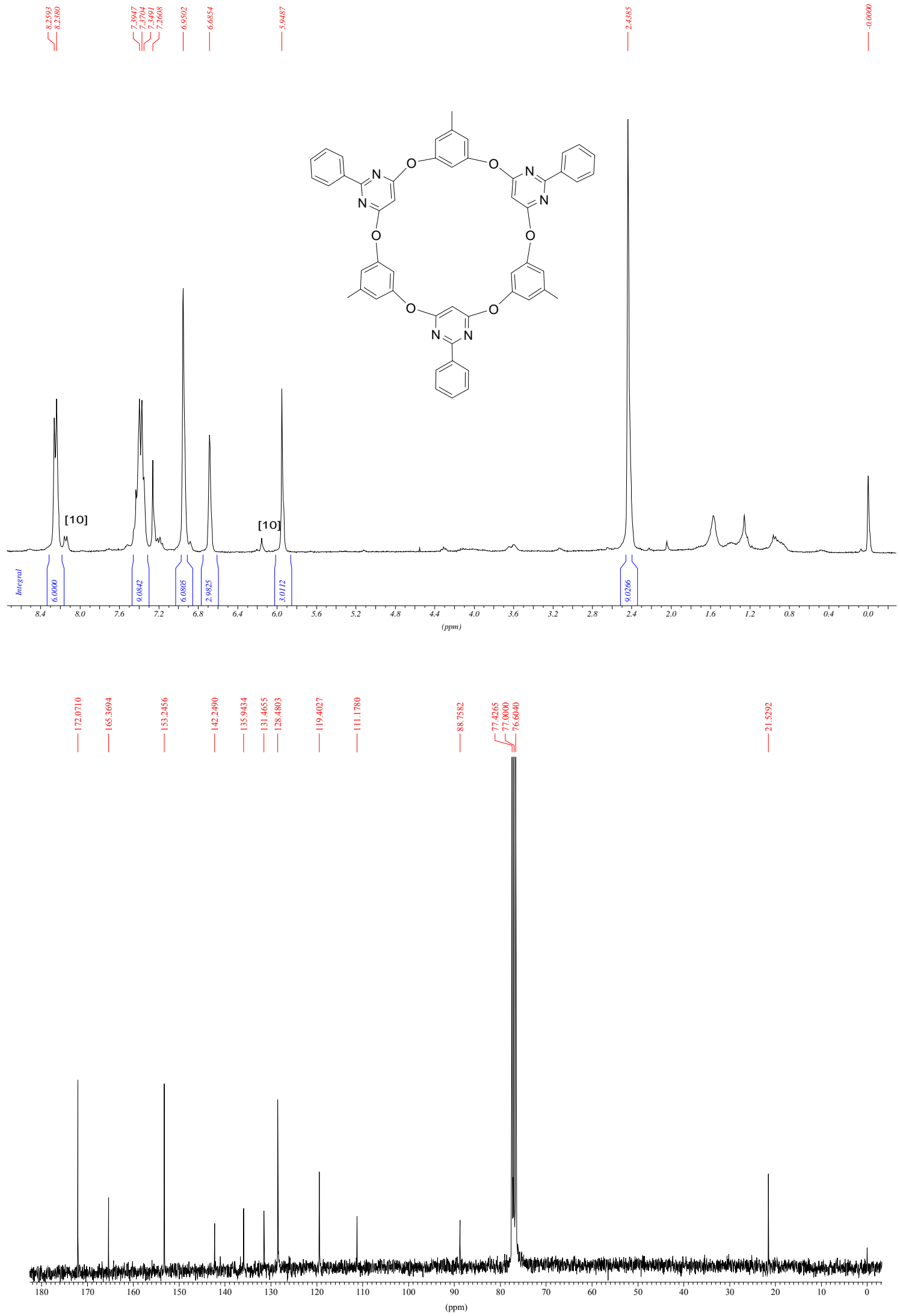
* Oxacalix[8]arene 5a
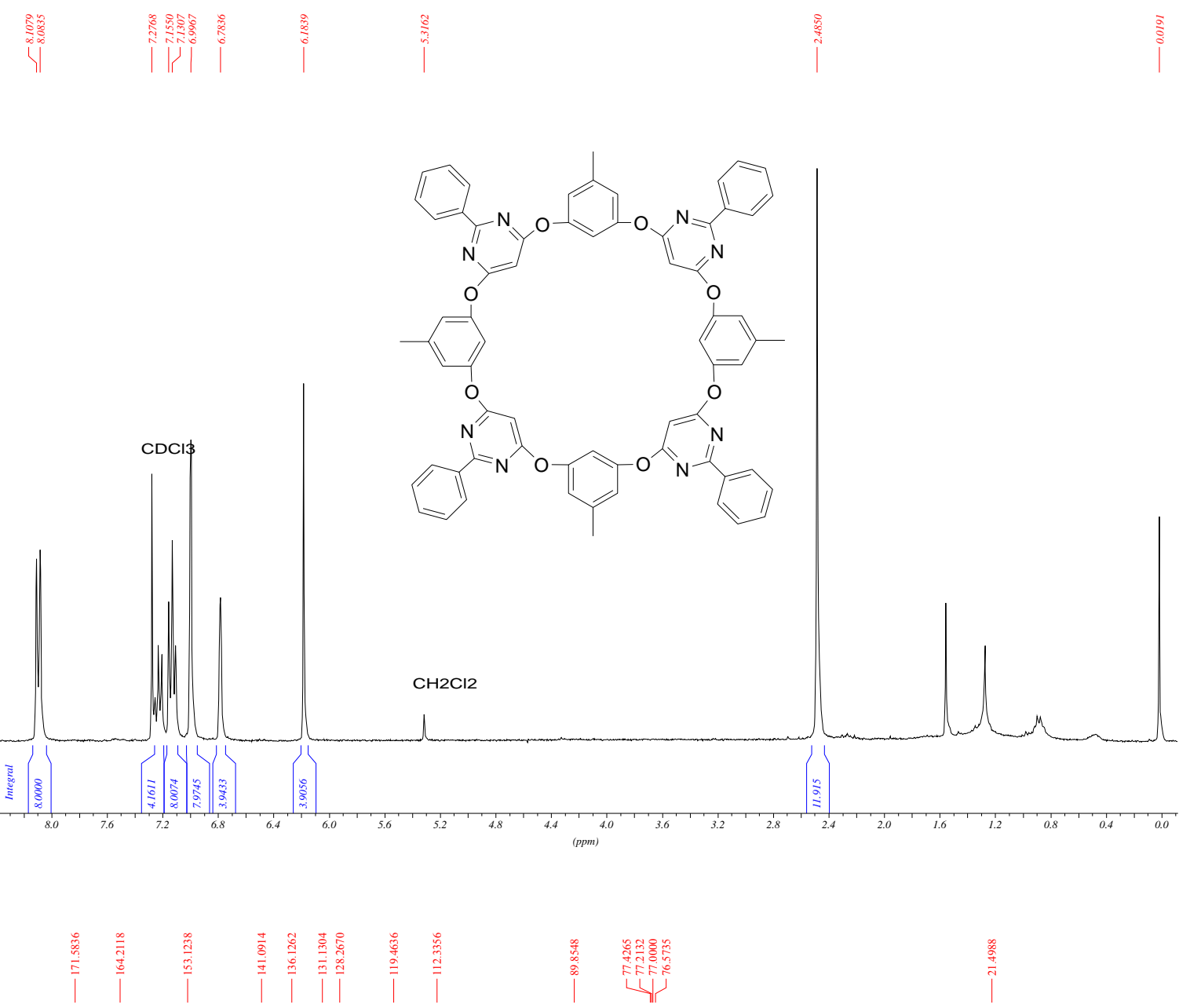
* Oxacalix[10]arene 6a
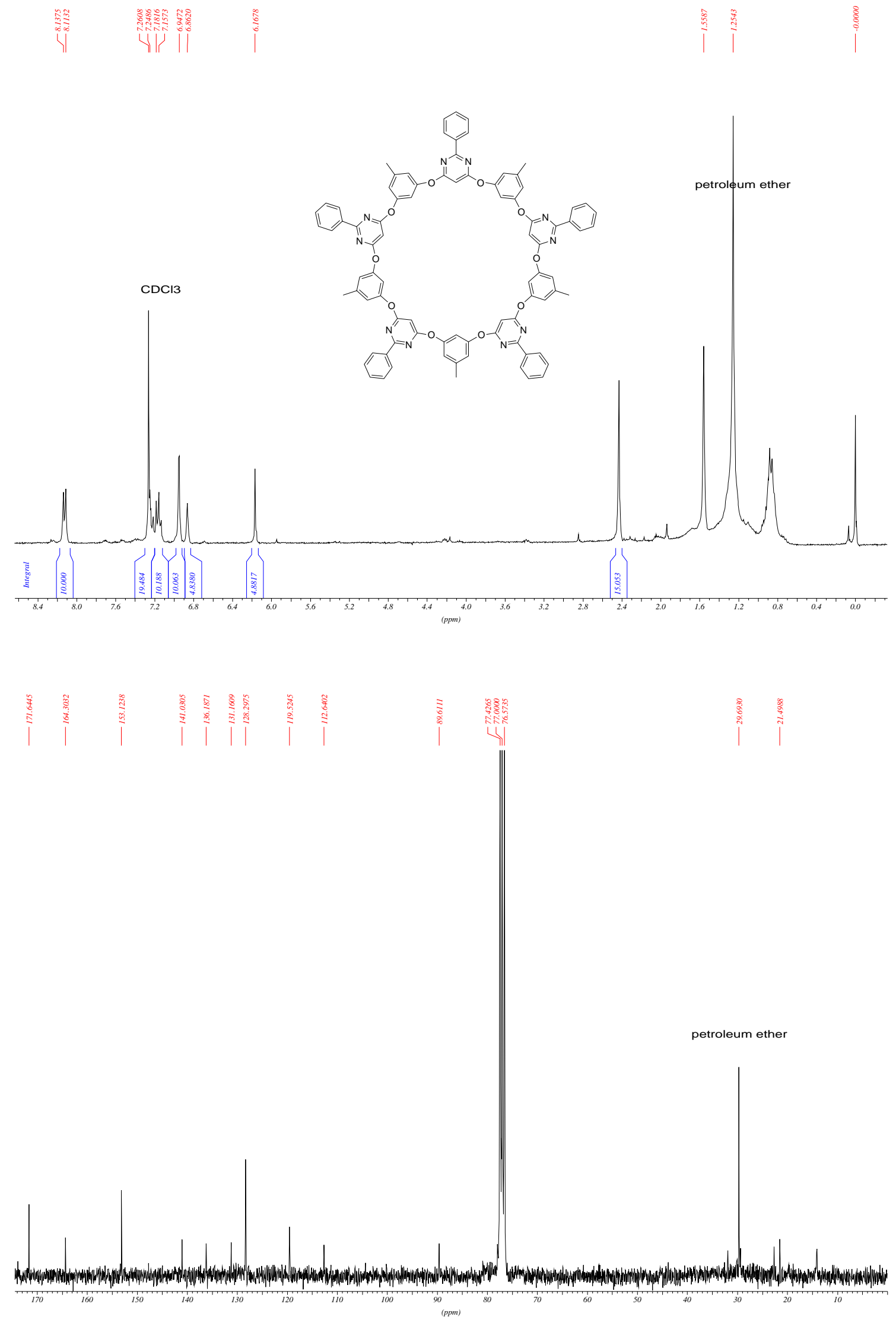
* Oxacalix[12]arene 7a
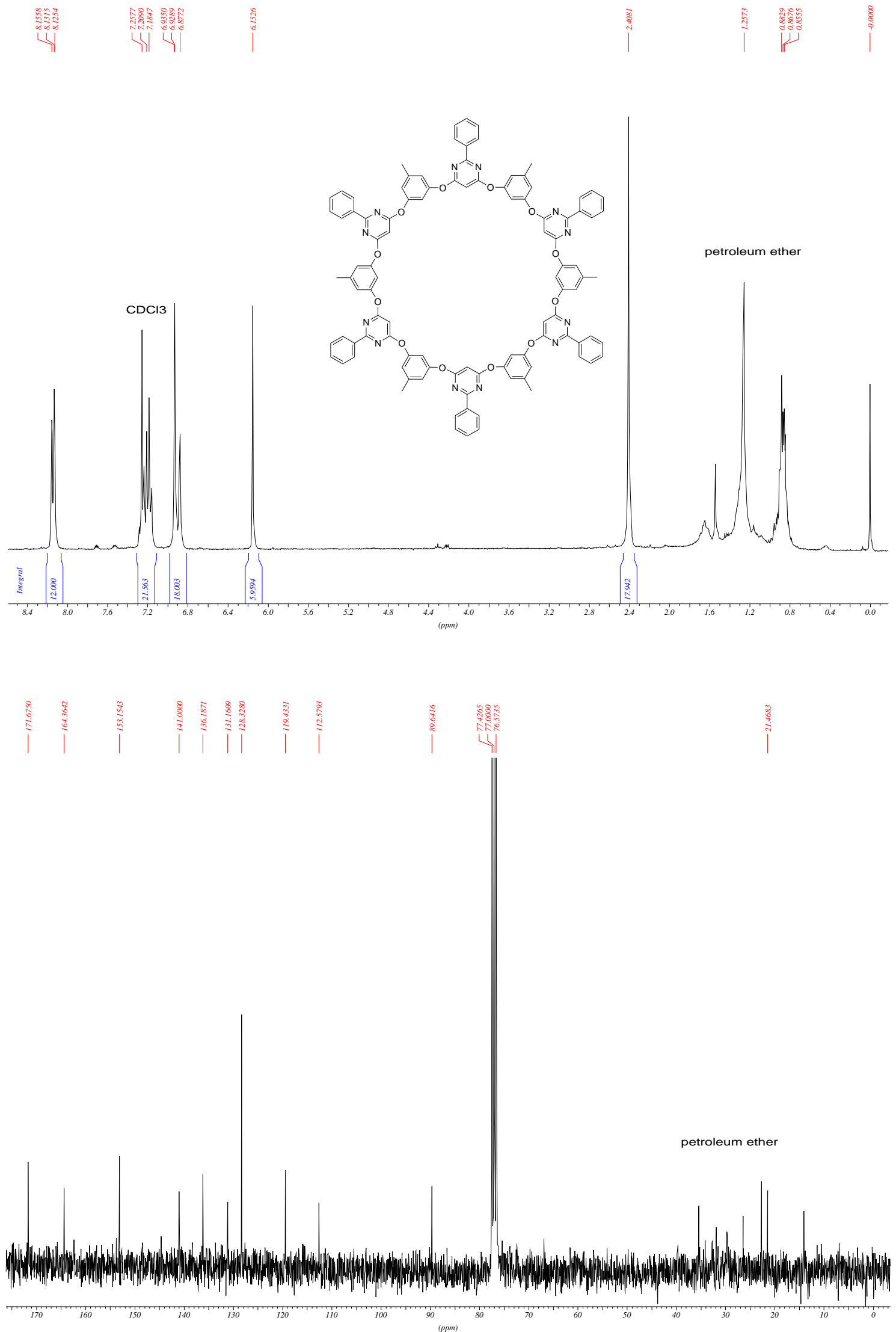
* Oxacalix[4]arene 3b

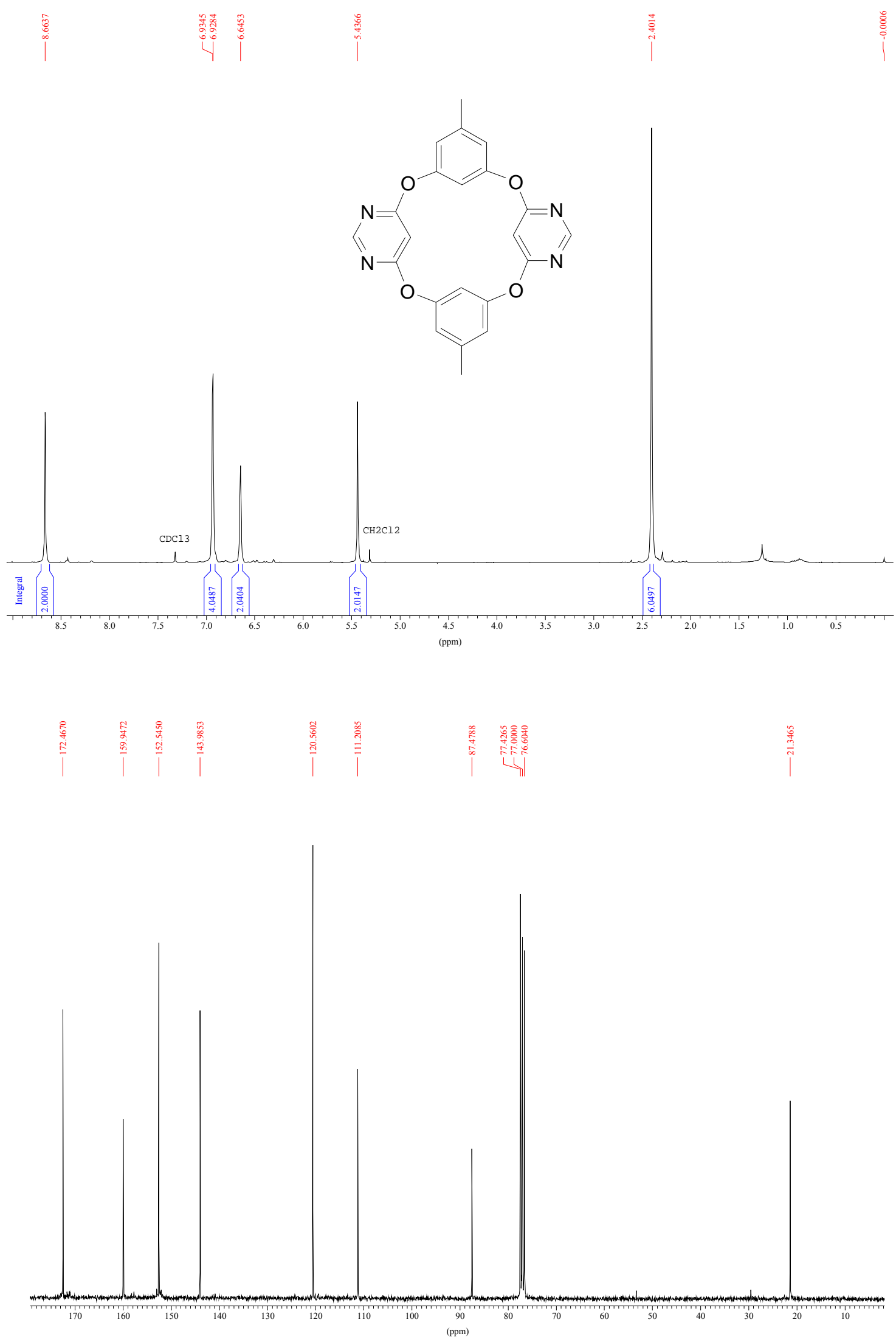


* Oxacalix[4]arene 3c
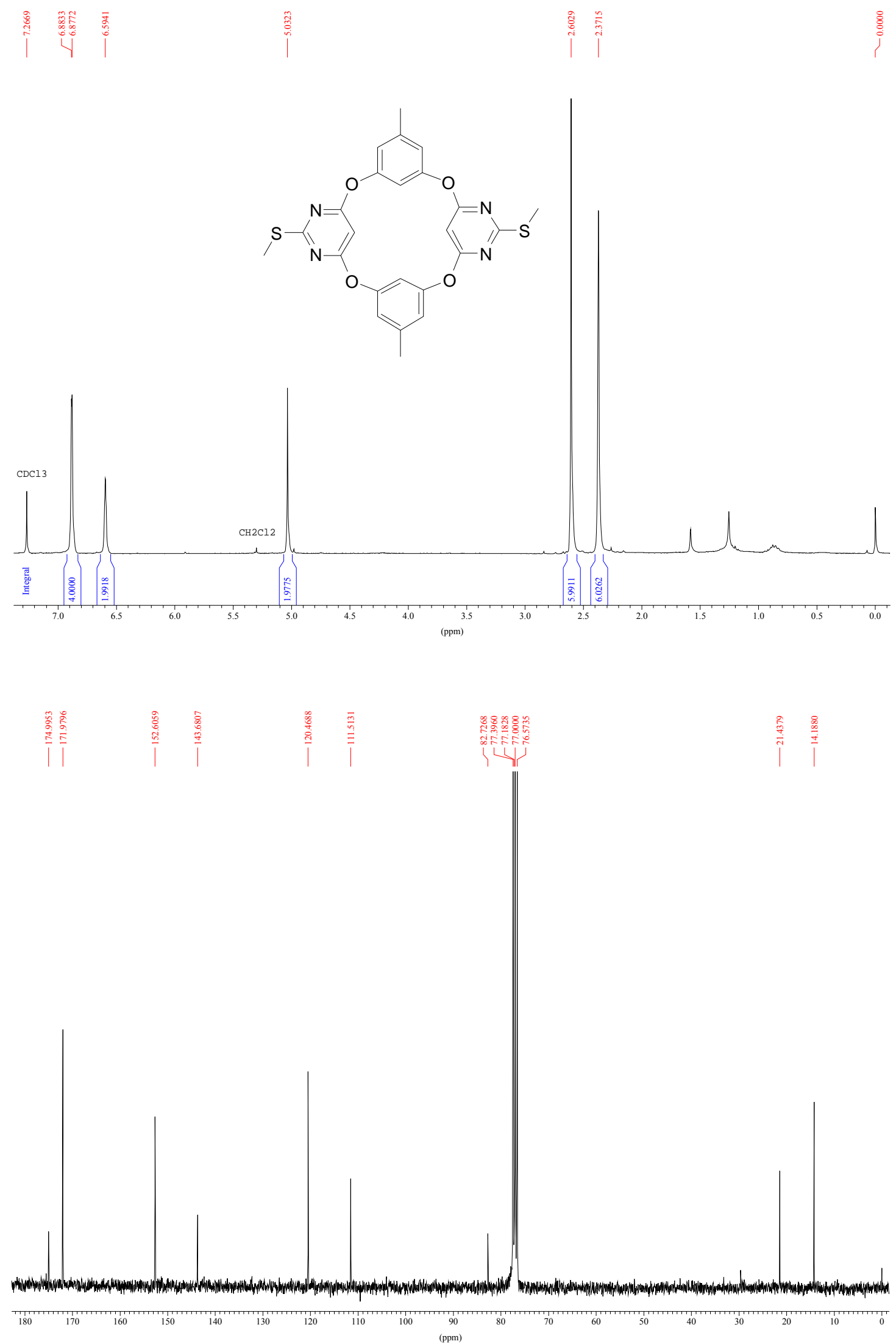
* Oxacalix[4]arene 3d

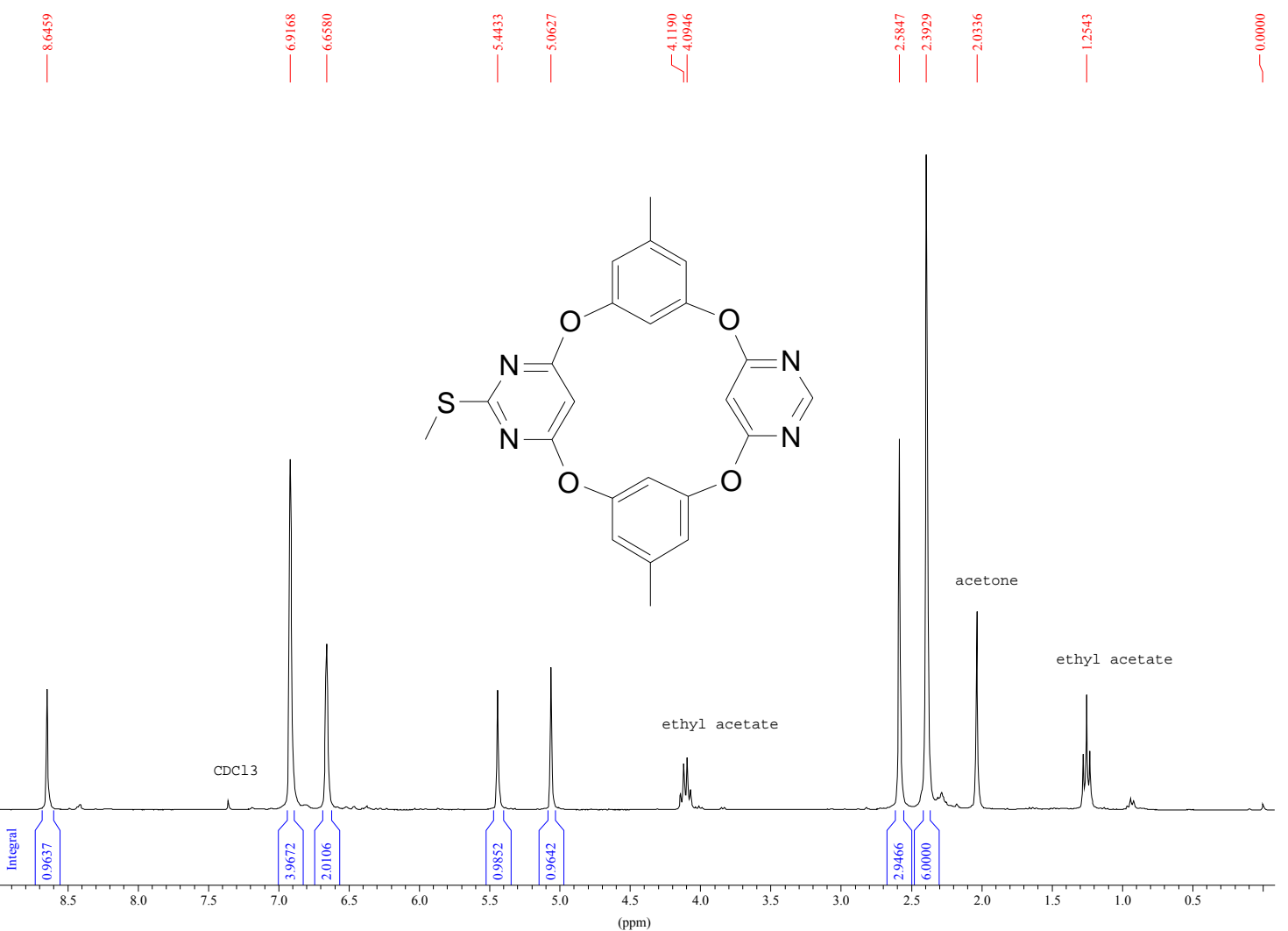

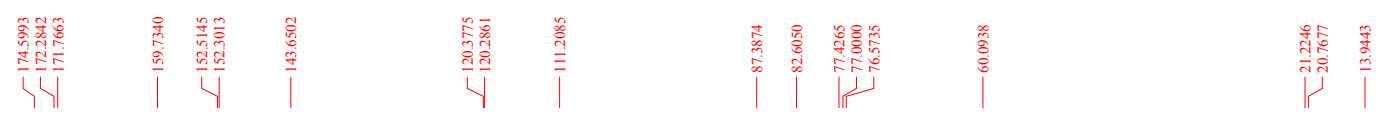

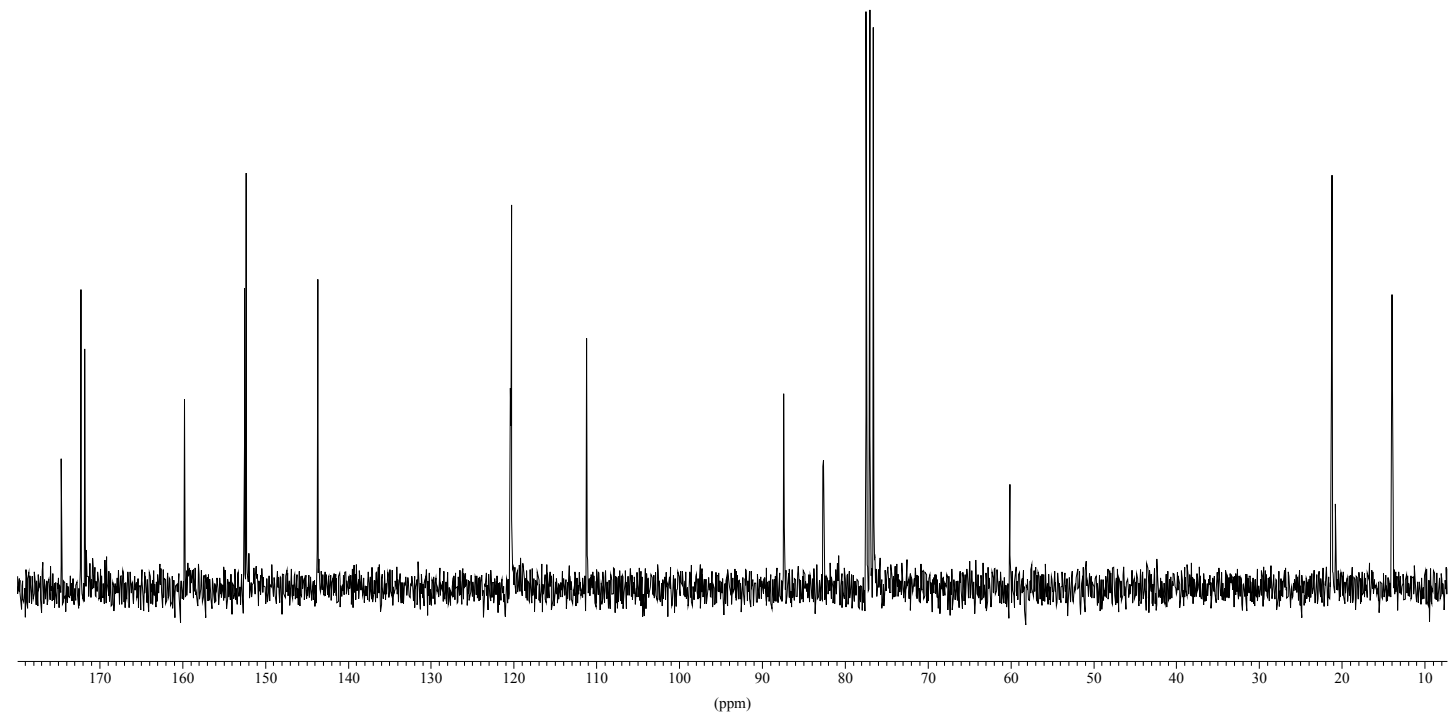


* Oxacalix[4]arene 3g

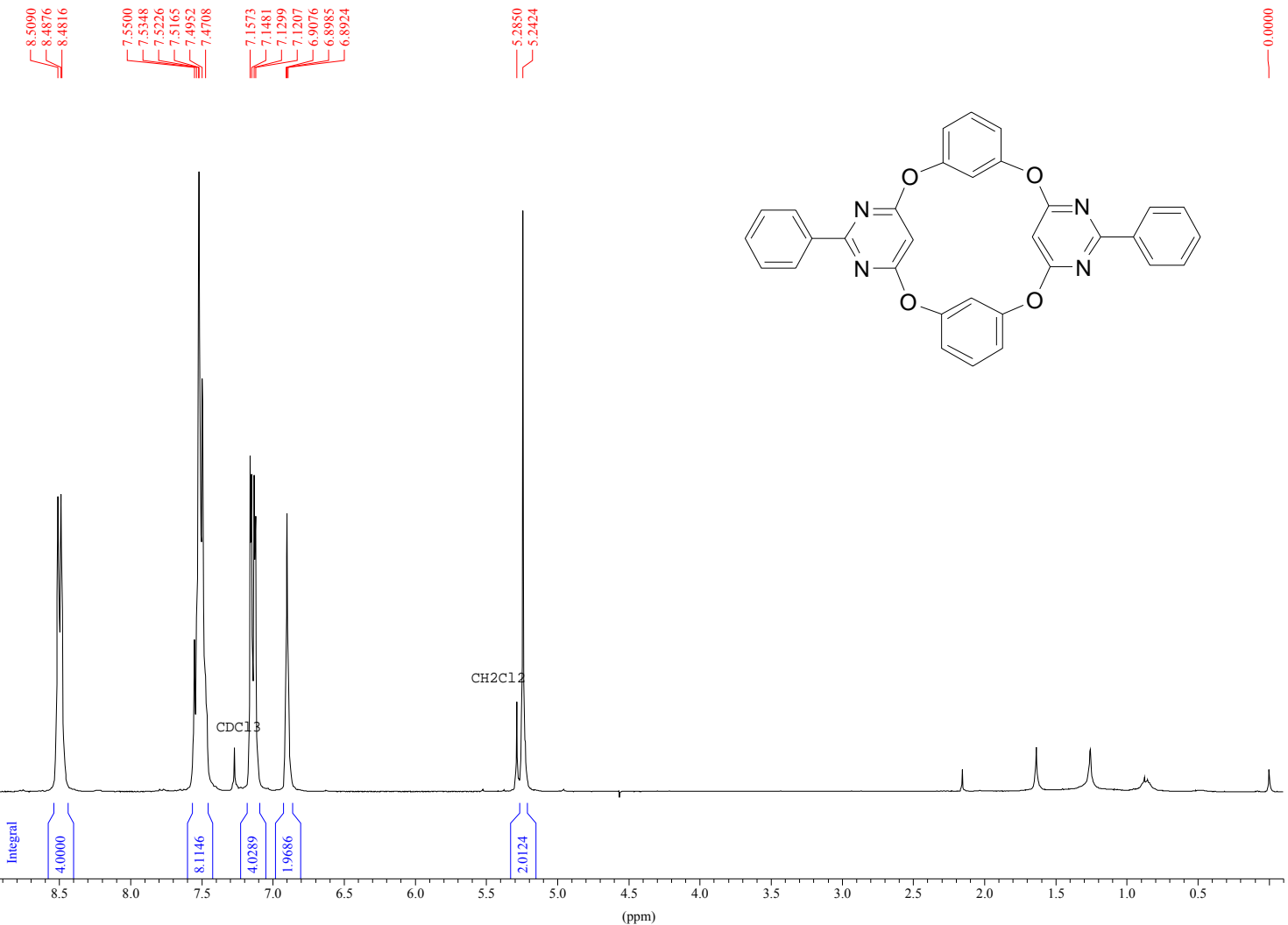

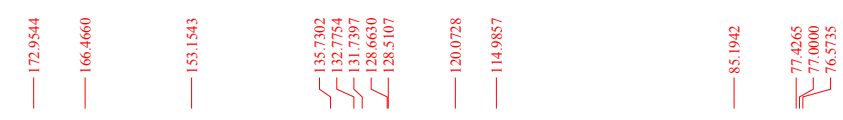

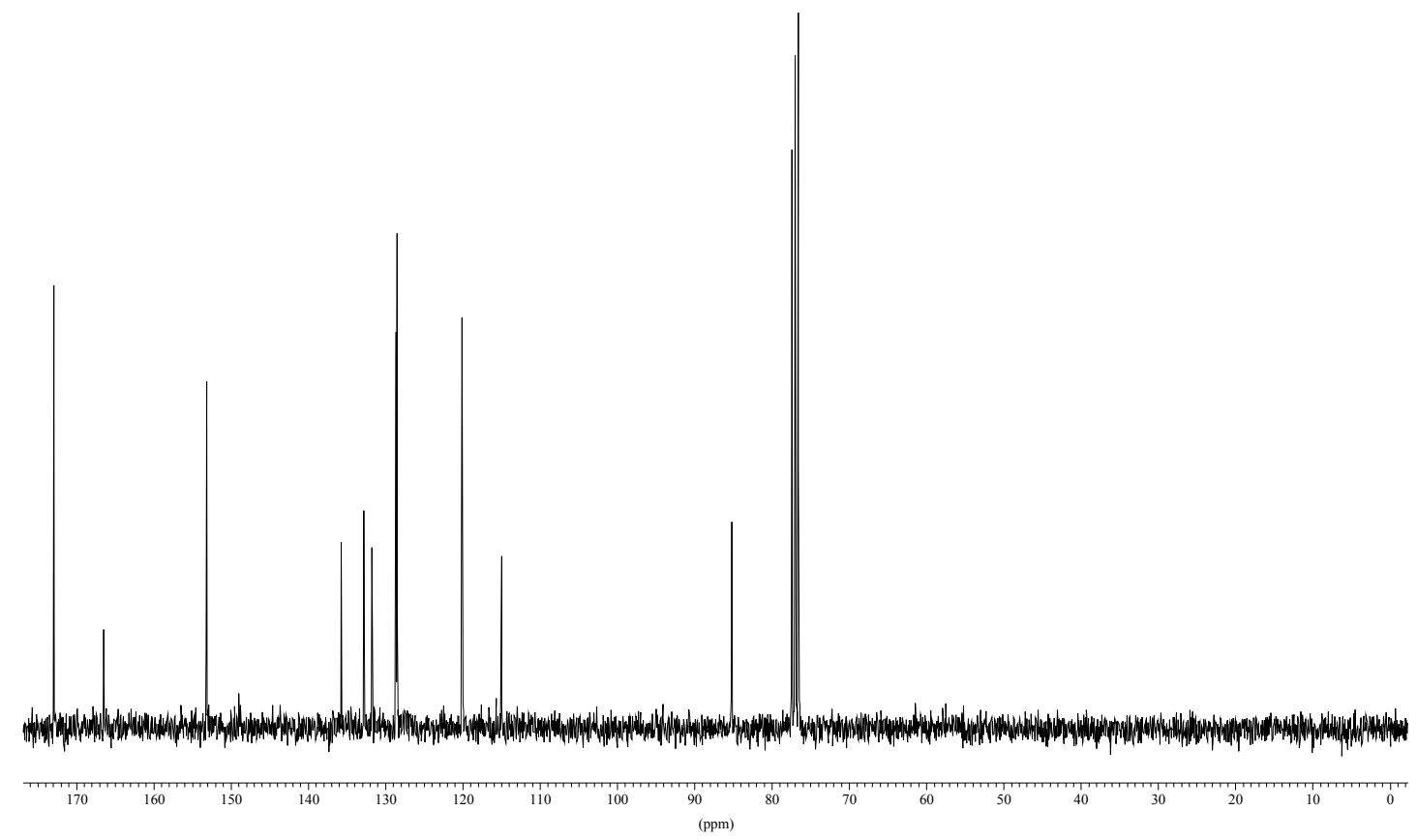


* Oxacalix[4] arene 3h (DMSO- $d_{6}-\mathrm{CDCl}_{3}$ 1-1)
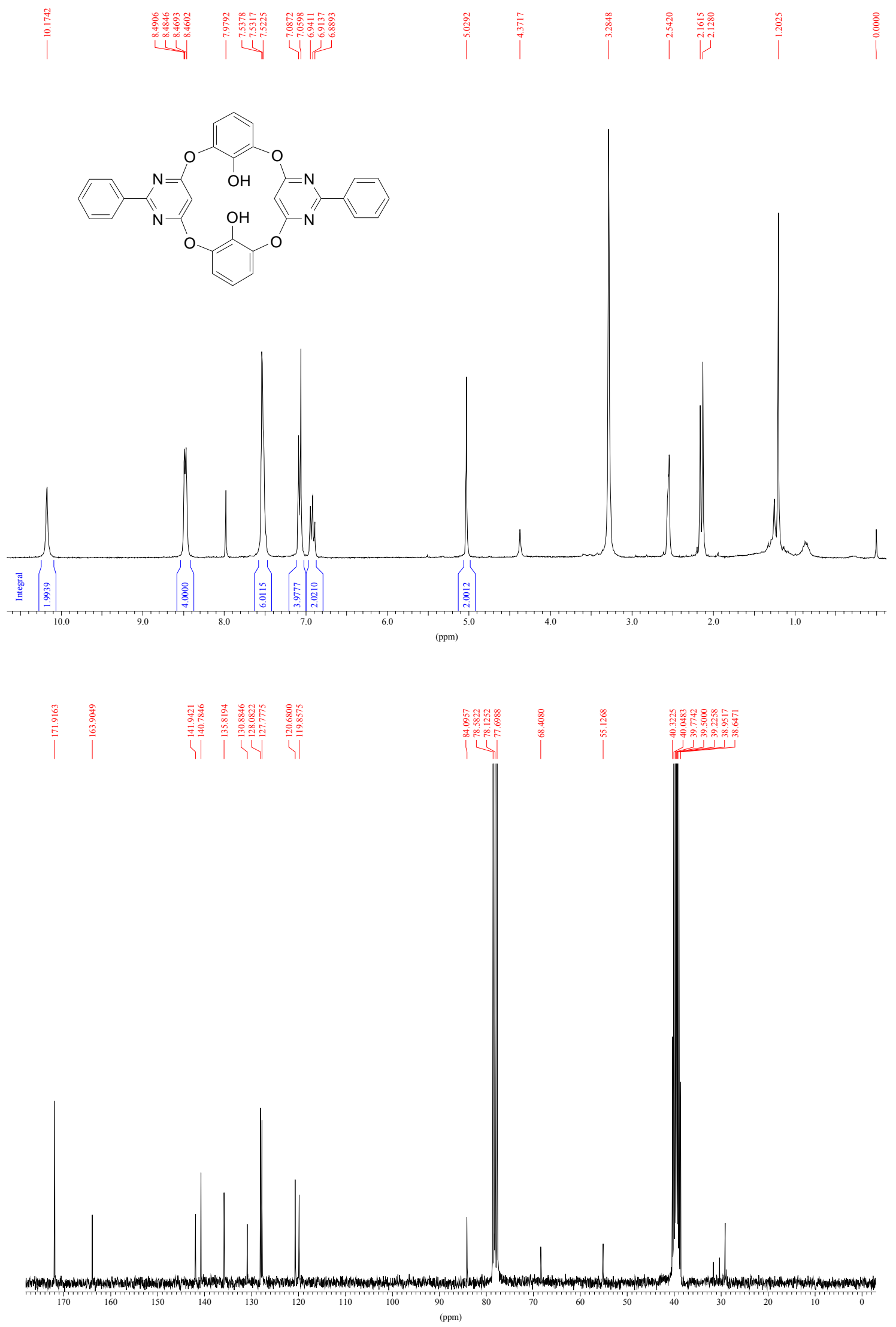
* Oxacalix[4]arene 3i

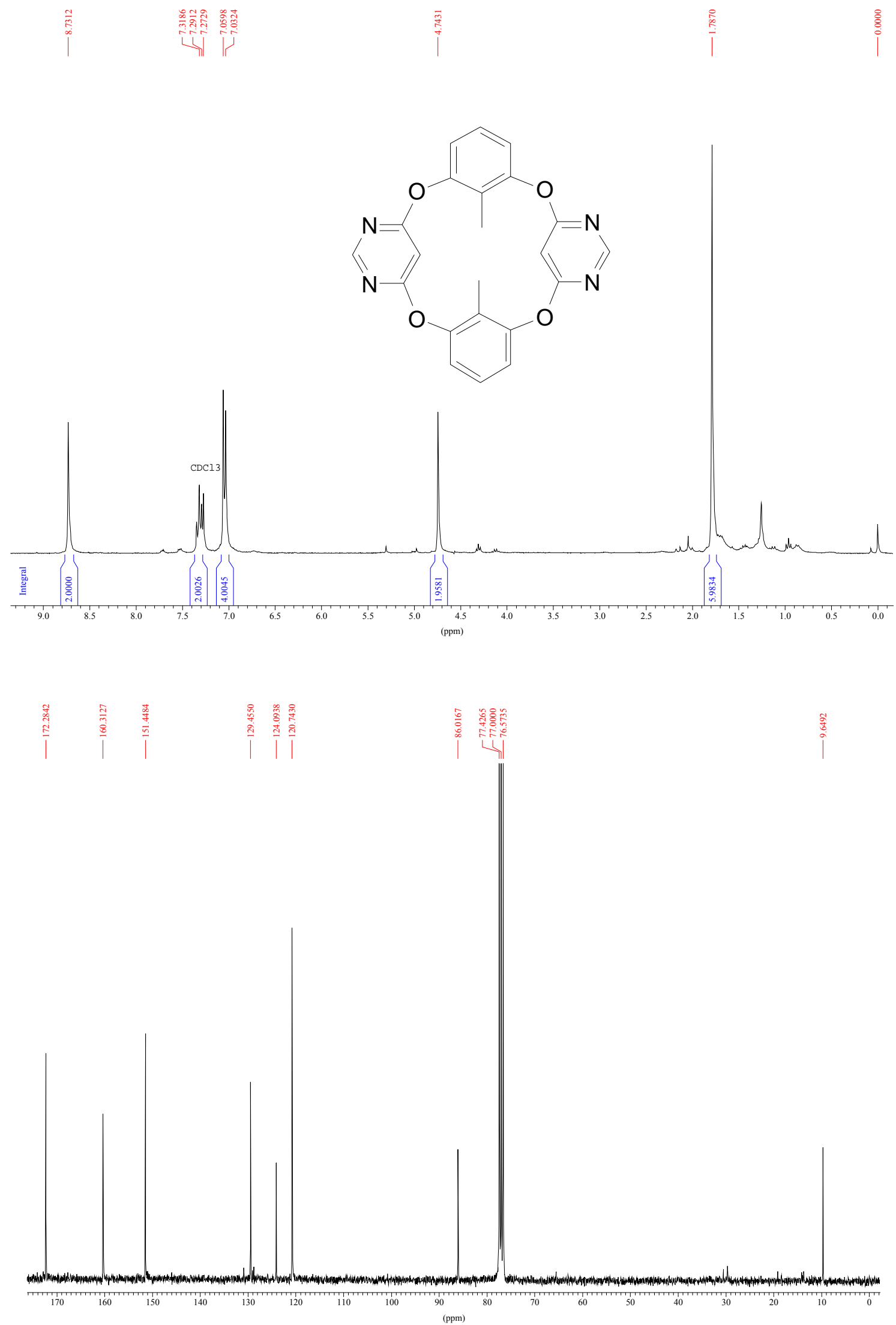


* Thiacalix[4]arene 8

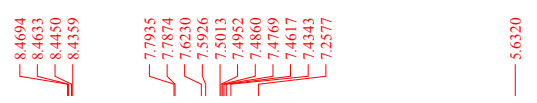
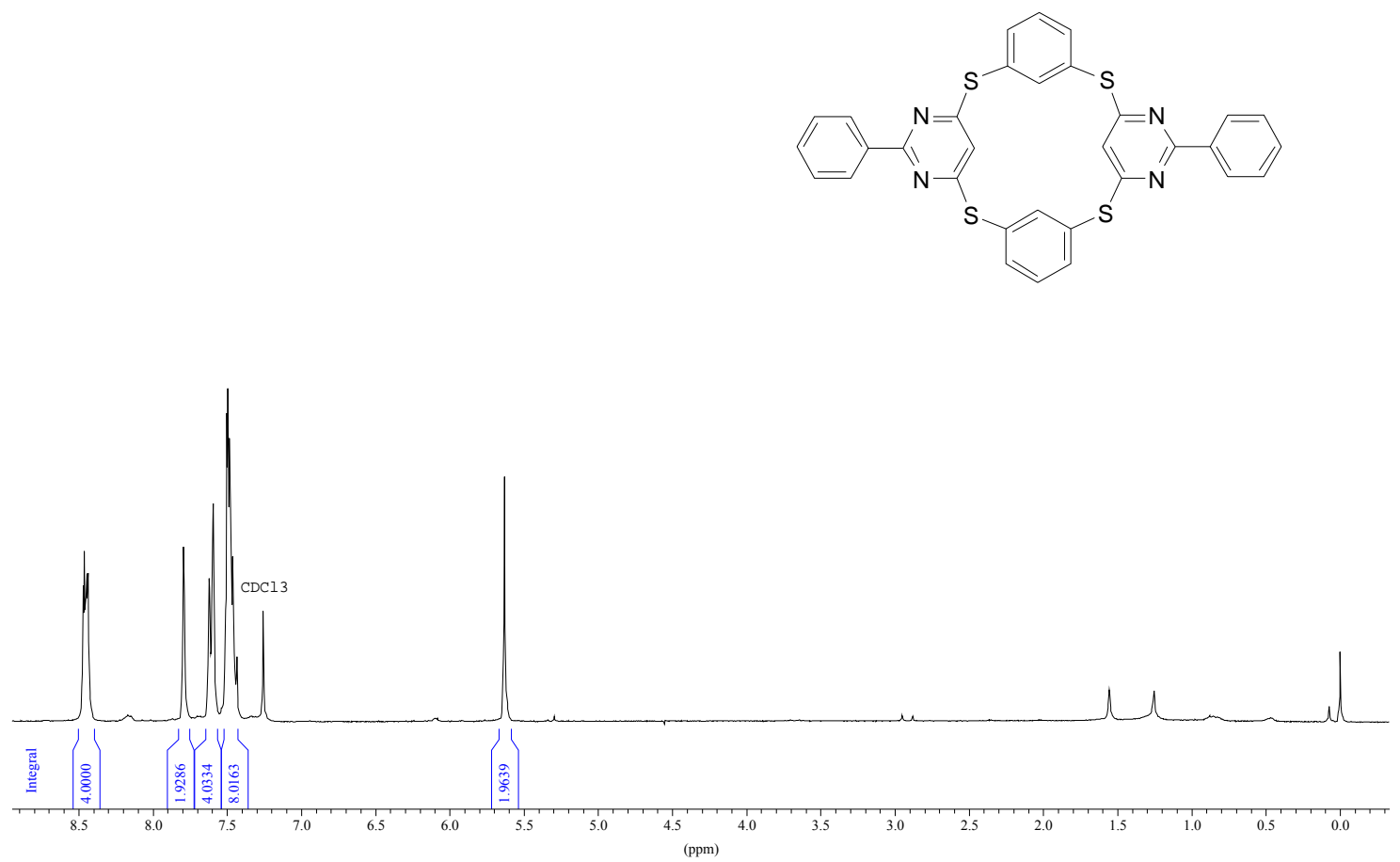

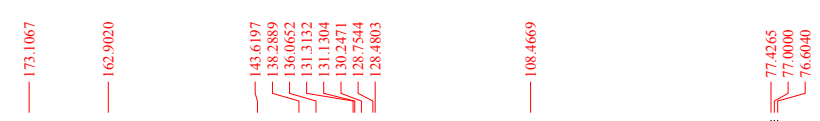

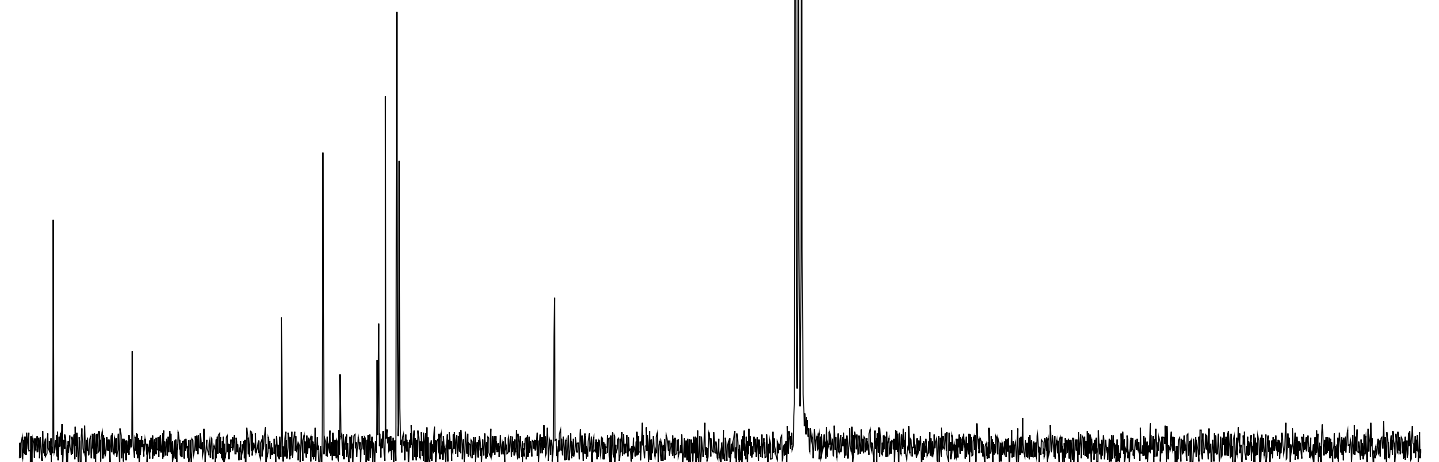

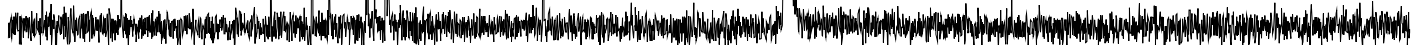

${ }_{170}{ }_{160}{ }_{150}$




\section{X-ray crystallographic structure and data for oxacalix[4]arene 3a}

Crystals of oxacalix[4]arene 3a, suitable for X-ray diffraction, were obtained by vapor diffusion of pentane into a $\mathrm{CHCl}_{3}$ solution of 3a (at $\mathrm{rt}$ ). A transparent, needle-shaped crystal with approximate dimensions of $0.6 \times 0.3 \times 0.2 \mathrm{~mm}$ was selected and mounted in a nylon loop for data collection. X-ray intensity data were collected at $100 \mathrm{~K}$ on a SMART 6000 diffractometer equipped with CCD detector using $\mathrm{CuK} \alpha$ radiation $(\lambda=1.54178 \AA)$, using phi and omega scans. The images were interpreted and integrated with the program SAINT from Bruker, ${ }^{1}$ giving a total of 11670 reflections (2447 independent reflections, $R_{\text {int }}=0.0326$ ). The structure was solved by direct methods and refined by full-matrix leastsquares on $\mathrm{F}^{2}$ using the SHELXTL program package, ${ }^{2}$ converging to a final $R=0.0410$ for 2214 reflections with $I>2 \sigma(I)$ and $\omega R 2=0.1107$ for all data and GOOF $=1.050$. Non-hydrogen atoms were anisotropically refined and the hydrogen atoms in the riding mode with isotropic temperature factors fixed at 1.2 times $U(\mathrm{eq})$ of the parent atoms and 1.5 times $U(\mathrm{eq})$ for methyl groups.

* Crystallographic data for the structure of oxacalix[4]arene 3a:

\begin{tabular}{|c|c|}
\hline $\begin{array}{l}\text { formula } \\
M\left(\mathrm{gmol}^{-1}\right)\end{array}$ & $\begin{array}{l}\mathrm{C}_{34} \mathrm{H}_{24} \mathrm{~N}_{4} \mathrm{O}_{4} \\
552.57\end{array}$ \\
\hline $\begin{array}{l}M\left(\mathrm{gmol}^{-}\right) \\
\text {crystal dimensions }\left(\mathrm{mm}^{3}\right)\end{array}$ & $0.6 \times 0.3 \times 0.2$ \\
\hline crystal system & monoclinic \\
\hline space group & $\mathrm{C} 2 / \mathrm{c}$ \\
\hline$a(\AA)$ & $33.2904(6)$ \\
\hline$b(\AA)$ & $4.64990(10)$ \\
\hline$c(\AA)$ & $21.8825(3)$ \\
\hline$\alpha(\mathrm{deg})$ & 90.000 \\
\hline$\beta(\operatorname{deg})$ & $129.9540(10)$ \\
\hline$\gamma(\mathrm{deg})$ & 90.000 \\
\hline$V / \AA^{30}$ & $2596.60(9)$ \\
\hline Z & 4 \\
\hline$\rho_{\text {calc }}\left(\mathrm{gcm}^{-3}\right)$ & 1.413 \\
\hline $2 \theta_{\max }\left({ }^{\circ}\right)$ & 141.72 \\
\hline radiation & $\mathrm{CuK \alpha}$ \\
\hline$\lambda(\AA)$ & 1.54178 \\
\hline$F(000)$ & 1151 \\
\hline$T(\mathrm{~K})$ & $100(2)$ \\
\hline measured reflections & 11670 \\
\hline unique reflections & 2447 \\
\hline observed reflections $\left(\mathrm{I}_{0}>2 \sigma\left(\mathrm{I}_{0}\right)\right)$ & 2214 \\
\hline parameters refined & 191 \\
\hline$R_{1}$ & 0.0410 \\
\hline$\omega R_{2}{ }^{\mathrm{a}}$ & 0.1072 \\
\hline$R_{1}$ (all data) & 0.0443 \\
\hline$\omega R_{2}$ (all data) & 0.1107 \\
\hline GOOF & 1.050 \\
\hline$\mu\left(\mathrm{mm}^{-1}\right)$ & 0.767 \\
\hline
\end{tabular}

${ }^{\mathrm{a}}$ Weighing scheme as defined for 3a: $\omega=1 /\left[\rho^{2}\left(F_{\mathrm{o}}{ }^{2}\right)+(0.0637 P)^{2}+2.0491 P\right], P=\left[\left(F_{\mathrm{o}}{ }^{2}+2 F_{\mathrm{c}}{ }^{2}\right) / 3\right]$.

CCDC-***** contains the supplementary crystallographic data for this paper. These data can be obtained free of charge via www.cam.ac.uk/conts/retrieving.html (or from the Cambridge Crystallographic Data Centre, 12 Union Road, Cambridge CB2 1EZ, UK; fax: (+44-1223-336033; or deposit@,ccdc.cam.uk).

${ }^{1}$ SAINT, Manual Version 5/6.0, Bruker Analytical X-ray Systems Inc.: Madison, Wisconsin, 1997.

2 SHELXTL-NT, Manual Version 5.1, Bruker Analytical X-ray Systems Inc.: Madison, Wisconsin, 1997. 


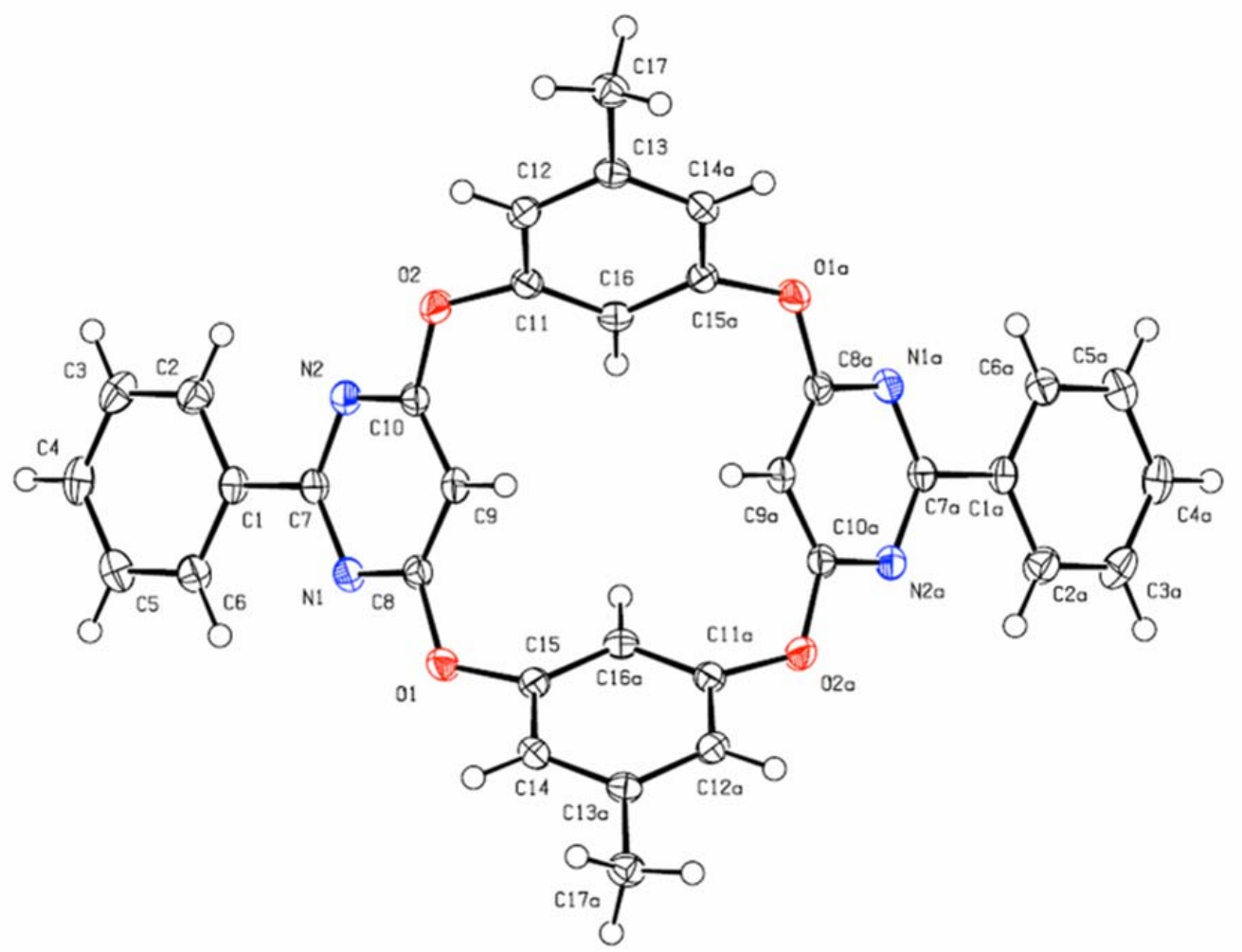

* Distances between the bridging O-atoms and the electrophilic (pyrimidine) and nucleophilic (benzene) component aromatic rings:

O-electrophilic component: average distance: $1.363 \AA$

O-nucleophilic component: average distance: $1.392 \AA$

Difference $\sim 0.03 \AA$

* Angles between the planes through the component aromatic rings:

C8 C9 C10 N2 C7 N1 and C8a C9a C10a N2a C7a N1a: 86.7

C11 C12 C13 C14a C15a C16 and C11a C12a C13a C14 C15 C16a: 86.1

* Distances between the centroids of the planes through the component aromatic rings:

C1 C2 C3 C4 C5 C6 and C1a C2a C3a C4a C5a C6a: $12.47 \AA$

C8 C9 C10 N2 C7 N1 and C8a C9a C10a N2a C7a N1a: $6.36 \AA$

C11 C12 C13 C14a C15a C16 and C11a C12a C13a C14 C15 C16a: $6.34 \AA$ 
* Crystal packing:

Along the b-axis, the formation of columns is observed, by stacking of the molecules of oxacalix[4]arene 3a. The molecules literally "hook" in each other.

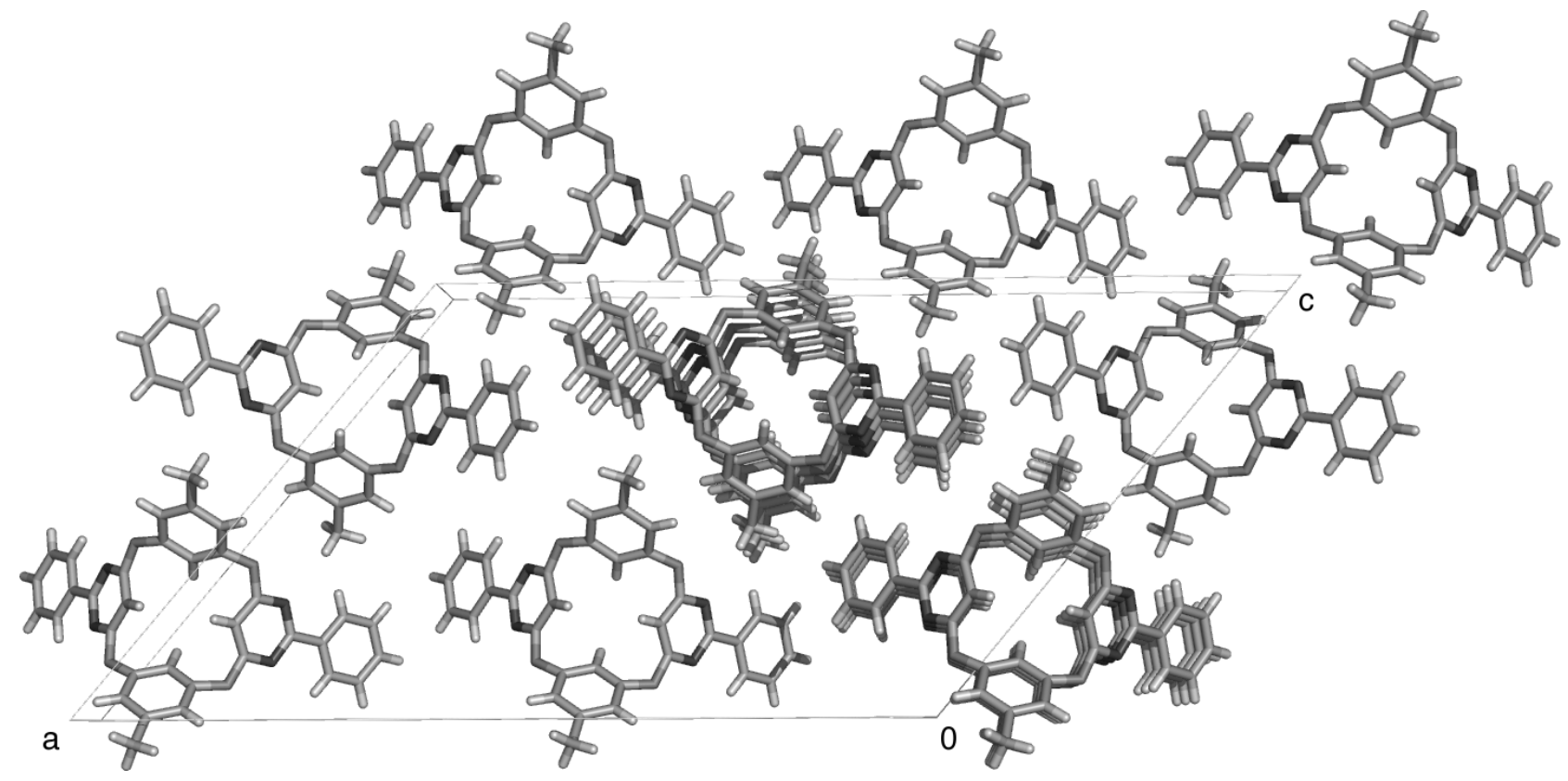

$\pi-\pi$ stacking occurs especially between the benzene ring of the electrophilic component and the (equiplanar) pyrimidine ring of the same electrophilic component of an understacked molecule (3.40 $\AA$ between planes through ring atoms).

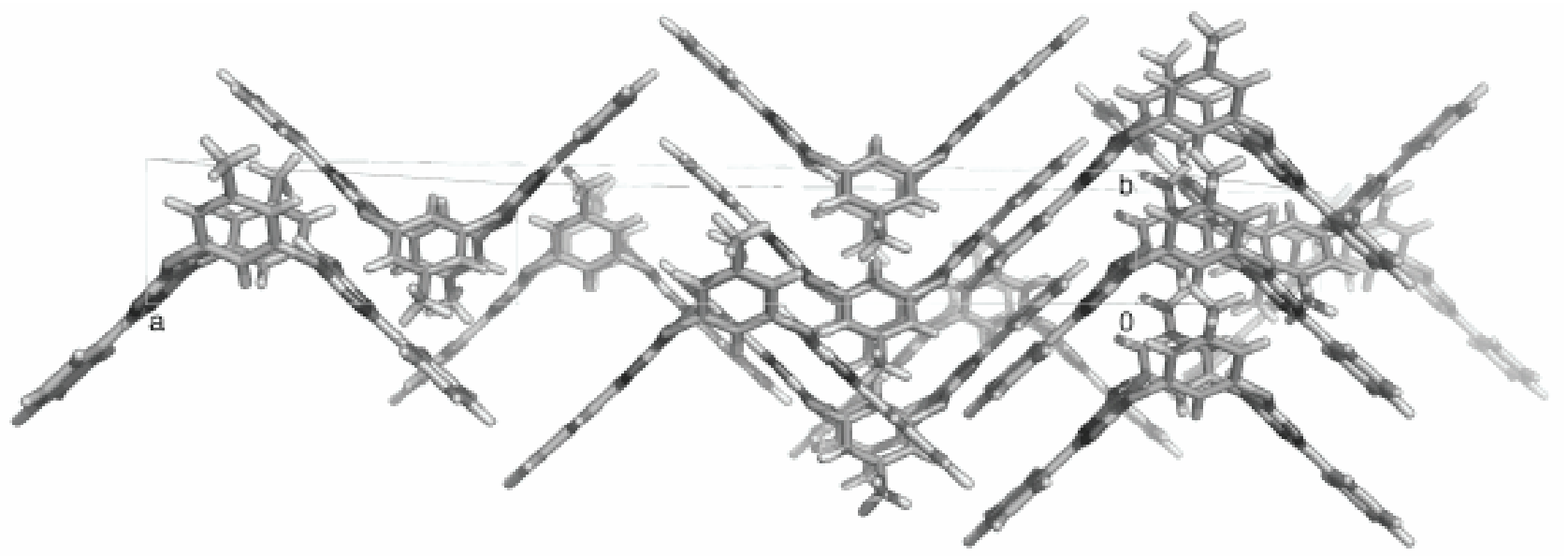

